\title{
Supersymmetric solutions to Euclidean Romans supergravity
}

\author{
Luis F. Alday, Martin Fluder, Carolina Matte Gregory, Paul Richmond \\ and James Sparks \\ Mathematical Institute, University of Oxford, Andrew Wiles Building, \\ Radcliffe Observatory Quarter, Woodstock Road, Oxford, OX2 6GG, U.K. \\ E-mail: luis.alday@maths.ox.ac.uk, fluder@caltech.edu, \\ mattegregory@maths.ox.ac.uk, paul.richmond@maths.ox.ac.uk, \\ james.sparks@maths.ox.ac.uk
}

ABSTRACT: We study Euclidean Romans supergravity in six dimensions with a non-trivial Abelian R-symmetry gauge field. We show that supersymmetric solutions are in one-to-one correspondence with solutions to a set of differential constraints on an SU(2) structure. As an application of our results we (i) show that this structure reduces at a conformal boundary to the five-dimensional rigid supersymmetric geometry previously studied by the authors, (ii) find a general expression for the holographic dual of the VEV of a BPS Wilson loop, matching an exact field theory computation, (iii) construct holographic duals to squashed Sasaki-Einstein backgrounds, again matching to a field theory computation, and (iv) find new analytic solutions.

KEYWORDS: Supersymmetric gauge theory, Gauge-gravity correspondence, Supergravity Models

ARXiv EPrint: 1505.04641 


\section{Contents}

1 Introduction $\quad 1$

2 Conditions for supersymmetry 2

2.1 Euclidean Romans supergravity 2

$2.2 \mathrm{SU}(2)$ structure 3

2.3 Differential constraints 4

2.4 Sufficiency 6

$\begin{array}{lll}2.5 & \text { Summary } & 7\end{array}$

3 Applications $\quad 8$

3.1 Expansion at a conformal boundary 8

3.2 BPS Wilson loops 9

$\begin{array}{ll}3.3 & \text { Squashed Sasaki-Einstein solutions } \\ & 12\end{array}$

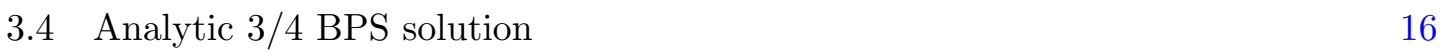

$\begin{array}{lll}4 & \text { Discussion } & 19\end{array}$

$\begin{array}{lr}\text { A Useful identities } & 19\end{array}$

$\begin{array}{ll}\text { B Differential conditions for bilinears } & 20\end{array}$

$\begin{array}{ll}\text { C More on the dilatino equation } & 23\end{array}$

$\begin{array}{ll}\text { D Integrability conditions } & 24\end{array}$

E Supersymmetry of the fundamental string $\quad 25$

\section{Introduction}

Advances in localization techniques applied to gauge theories have led to exact results for supersymmetric observables on general backgrounds. In three and four dimensions it turns out that such observables depend on only a small number of parameters of the full parameter space of the background [1,2]. Rigid supersymmetric gauge theories in five-dimensional curved backgrounds have been constructed and studied in a series of papers [3-17]. In the approach of [15] these rigid backgrounds are equipped with a transversely holomorphic foliation. Inspired by the lower-dimensional results of $[1,2]$ it was conjectured that supersymmetric observables depend only on this foliation. In this paper we systematically study supersymmetric solutions to Euclidean Romans supergravity in six dimensions. Our aim is 
to compute observables of interest for gauge/gravity duality, and in particular understand the conjecture of [15] from a holographic perspective.

Our starting point is to show that real Euclidean supersymmetric solutions to Romans $F(4)$ gauged supergravity, with a non-trivial Abelian R-symmetry gauge field, have a canonical SU(2) structure determined by the Killing spinor. More precisely we show that supersymmetry together with the equations of motion are equivalent to a set of differential constraints on this $\mathrm{SU}(2)$ structure. This geometric formulation then leads to a number of interesting applications. First, we show that this structure extends into the bulk the conformal boundary SU(2) structure studied in [15]. This allows for the construction of gravity duals to families of five-dimensional gauge theories on rigid backgrounds. As another application we extend several of the results in $[18,19]$. In the latter we constructed supergravity solutions with squashed five-sphere boundaries, and computed the holographic free energy and certain BPS Wilson loops. In the present paper we extend these results to new families of solutions, in general with different topology. In particular this includes squashed Sasaki-Einstein conformal boundaries, together with new analytic solutions. Furthermore, in $[18,19]$ we conjectured a general formula for the VEV of a BPS Wilson loop, both in field theory and in supergravity. In this paper the supergravity conjecture is proven.

The outline of the paper is as follows. Section 2 contains a general analysis of Euclidean supersymmetric solutions to Romans supergravity, recasting the conditions in terms of a canonical local SU(2) structure. In section 3 we present a number of applications of our formalism. Our conclusions are presented in section 4. A number of technical details have been included in five appendices.

\section{Conditions for supersymmetry}

\subsection{Euclidean Romans supergravity}

The bosonic fields of the six-dimensional Romans supergravity theory [20] consist of the metric, a scalar field $X=\exp \left(-\frac{\phi}{2 \sqrt{2}}\right)$ where $\phi$ is the dilaton, a two-form potential $B$, together with an $\mathrm{SO}(3)_{R} \sim \mathrm{SU}(2)_{R}$ R-symmetry gauge field $A^{i}$ with field strength $F^{i}=$ $\mathrm{d} A^{i}-\frac{1}{2} \varepsilon_{i j k} A^{j} \wedge A^{k}$, where $i=1,2,3$. Here we are working in a gauge in which the Stueckelberg one-form is zero, and we set the gauge coupling constant to 1 . The Euclidean signature equations of motion are [19]

$$
\begin{aligned}
\mathrm{d}\left(X^{-1} * \mathrm{~d} X\right)= & -\left(\frac{1}{6} X^{-6}-\frac{2}{3} X^{-2}+\frac{1}{2} X^{2}\right) * 1 \\
& -\frac{1}{8} X^{-2}\left(\frac{4}{9} B \wedge * B+F^{i} \wedge * F^{i}\right)+\frac{1}{4} X^{4} H \wedge * H, \\
\mathrm{~d}\left(X^{4} * H\right)= & \frac{2 \mathrm{i}}{9} B \wedge B+\frac{\mathrm{i}}{2} F^{i} \wedge F^{i}+\frac{4}{9} X^{-2} * B, \\
D\left(X^{-2} * F^{i}\right)= & -\mathrm{i} F^{i} \wedge H .
\end{aligned}
$$

Here $H=\mathrm{d} B$ and $D \omega^{i}=\mathrm{d} \omega^{i}-\varepsilon_{i j k} A^{j} \wedge \omega^{k}$ is the $\mathrm{SO}(3)$ covariant derivative. Notice that the theory contains Chern-Simons-type couplings, that become purely imaginary in 
Euclidean signature. The Einstein equation is

$$
\begin{aligned}
R_{\mu \nu}= & 4 X^{-2} \partial_{\mu} X \partial_{\nu} X+\left(\frac{1}{18} X^{-6}-\frac{2}{3} X^{-2}-\frac{1}{2} X^{2}\right) g_{\mu \nu}+\frac{1}{4} X^{4}\left(H_{\mu \nu}^{2}-\frac{1}{6} H^{2} g_{\mu \nu}\right) \\
& +\frac{2}{9} X^{-2}\left(B_{\mu \nu}^{2}-\frac{1}{8} B^{2} g_{\mu \nu}\right)+\frac{1}{2} X^{-2}\left(\left(F^{i}\right)_{\mu \nu}^{2}-\frac{1}{8}\left(F^{i}\right)^{2} g_{\mu \nu}\right),
\end{aligned}
$$

where $B_{\mu \nu}^{2}=B_{\mu \rho} B_{\nu}^{\rho}, H_{\mu \nu}^{2}=H_{\mu \rho \sigma} H_{\nu}^{\rho \sigma}$.

A solution is supersymmetric provided there exists a non-trivial $\mathrm{SU}(2)_{R}$ doublet of Dirac spinors $\epsilon_{I}, I=1,2$, satisfying the following Killing spinor and dilatino equations

$$
\begin{aligned}
D_{\mu} \epsilon_{I}= & \frac{\mathrm{i}}{4 \sqrt{2}}\left(X+\frac{1}{3} X^{-3}\right) \Gamma_{\mu} \Gamma_{7} \epsilon_{I}-\frac{\mathrm{i}}{24 \sqrt{2}} X^{-1} B_{\nu \rho}\left(\Gamma_{\mu}{ }^{\nu \rho}-6 \delta_{\mu}{ }^{\nu} \Gamma^{\rho}\right) \epsilon_{I} \\
& -\frac{1}{48} X^{2} H_{\nu \rho \sigma} \Gamma^{\nu \rho \sigma} \Gamma_{\mu} \Gamma_{7} \epsilon_{I}+\frac{1}{16 \sqrt{2}} X^{-1} F_{\nu \rho}^{i}\left(\Gamma_{\mu}^{\nu \rho}-6 \delta_{\mu}{ }^{\nu} \Gamma^{\rho}\right) \Gamma_{7}\left(\sigma_{i}\right)_{I}{ }^{J} \epsilon_{J}, \\
0= & -\mathrm{i} X^{-1} \partial_{\mu} X \Gamma^{\mu} \epsilon_{I}+\frac{1}{2 \sqrt{2}}\left(X-X^{-3}\right) \Gamma_{7} \epsilon_{I}+\frac{\mathrm{i}}{24} X^{2} H_{\mu \nu \rho} \Gamma^{\mu \nu \rho} \Gamma_{7} \epsilon_{I} \\
& -\frac{1}{12 \sqrt{2}} X^{-1} B_{\mu \nu} \Gamma^{\mu \nu} \epsilon_{I}-\frac{\mathrm{i}}{8 \sqrt{2}} X^{-1} F_{\mu \nu}^{i} \Gamma^{\mu \nu} \Gamma_{7}\left(\sigma_{i}\right)_{I}^{J} \epsilon_{J} .
\end{aligned}
$$

Here $\Gamma_{\mu}, \mu=1, \ldots, 6$, are taken to be Hermitian and generate the Clifford algebra Cliff $(6,0)$ in an orthonormal frame. We have defined the chirality operator $\Gamma_{7}=\mathrm{i} \Gamma_{123456}$, which satisfies $\left(\Gamma_{7}\right)^{2}=1$. The covariant derivative acting on the spinor is $D_{\mu} \epsilon_{I}=\nabla_{\mu} \epsilon_{I}+$ $\frac{\mathrm{i}}{2} A_{\mu}^{i}\left(\sigma_{i}\right)_{I}{ }^{J} \epsilon_{J}$, where $\nabla_{\mu}=\partial_{\mu}+\frac{1}{4} \Omega_{\mu}{ }^{\nu \rho} \Gamma_{\nu \rho}$ denotes the Levi-Civita spin connection while $\sigma_{i}$, $i=1,2,3$, are the Pauli matrices.

For simplicity we shall consider Abelian solutions in which $A_{\mu}^{1}=A_{\mu}^{2}=0$, and $A_{\mu}^{3} \equiv \mathcal{A}_{\mu}$, with field strength $\mathcal{F} \equiv \mathrm{d} \mathcal{A}$. Also, as in [19], we consider a "real" class of solutions for which $\epsilon_{I}$ satisfies the symplectic Majorana condition $\varepsilon_{I}^{J} \epsilon_{J}=\mathcal{C} \epsilon_{I}^{*} \equiv \epsilon_{I}^{c}$, where $\mathcal{C}$ denotes the charge conjugation matrix, satisfying $\Gamma_{\mu}^{\mathrm{T}}=\mathcal{C}^{-1} \Gamma_{\mu} \mathcal{C}$. The bosonic fields are all taken to be real, with the exception of the $B$-field which is purely imaginary. With these reality properties one can show that the Killing spinor equation (2.3) and dilatino equation (2.4) for $\epsilon_{2}$ are simply the charge conjugates of the corresponding equations for $\epsilon_{1}$. In this way we effectively reduce to a single Killing spinor $\epsilon \equiv \epsilon_{1}$, with $\operatorname{SU}(2)_{R} \operatorname{doublet}\left(\epsilon_{1}, \epsilon_{2}\right)=\left(\epsilon, \epsilon^{c}\right)$.

\section{$2.2 \mathrm{SU}(2)$ structure}

Consider a Dirac spinor $\epsilon$ in six dimensions, such that $\left(\epsilon_{1}, \epsilon_{2}\right)=\left(\epsilon, \epsilon^{c}\right)$ solves (2.3) and (2.4) above. We may construct the following scalar bilinears

$$
S \equiv \epsilon^{\dagger} \epsilon, \quad \tilde{S} \equiv \epsilon^{\dagger} \Gamma_{7} \epsilon, \quad f \equiv \epsilon^{\mathrm{T}} \epsilon .
$$

Here we have chosen a basis for the gamma matrices in which they are purely imaginary and anti-symmetric, with charge conjugation matrix $\mathcal{C}=-\mathrm{i} \Gamma_{7}$. A short computation reveals that

$$
\mathrm{d}(X f)=-\mathrm{i}(X f) \mathcal{A}
$$


The integrability condition for this equation immediately implies $\mathcal{F}=\mathrm{d} \mathcal{A}=0$ unless $f \equiv 0$ (notice that $X$ is nowhere zero). We will henceforth restrict our analysis to the case $f \equiv 0$, which is necessary for a non-trivial R-symmetry gauge field. ${ }^{1}$

We may then write

$$
\epsilon=\epsilon_{+}+\epsilon_{-},
$$

where $-\Gamma_{7} \epsilon_{ \pm}= \pm \epsilon_{ \pm}$, and furthermore the condition $f \equiv 0$ allows us to introduce [21]

$$
\epsilon_{+}=\sqrt{S} \cos \vartheta \eta_{1}, \quad \epsilon_{-}=\sqrt{S} \sin \vartheta \eta_{2}^{*} .
$$

Here $\eta_{1}, \eta_{2}$ are two orthogonal unit norm chiral spinors, so that $\eta_{1}^{\dagger} \eta_{1}=\eta_{2}^{\dagger} \eta_{2}=1$ and $\eta_{2}^{\dagger} \eta_{1}=$ 0 . These each define a canonical SU(3) structure, and together determine a canonical SU(2) structure. Concretely, in six dimensions such a structure is specified by two one-forms $K_{1}$, $K_{2}$ and a triplet of two-forms $J_{i}, i=1,2,3$, given by

$$
\begin{aligned}
K_{1}-\mathrm{i} K_{2} & \equiv-\frac{1}{2} \varepsilon^{\alpha \beta} \eta_{\alpha}^{\mathrm{T}} \Gamma_{(1)} \eta_{\beta}, \\
J_{i} & \equiv-\frac{\mathrm{i}}{2} \sigma_{i}^{\alpha \beta} \eta_{\alpha}^{\dagger} \Gamma_{(2)} \eta_{\beta} .
\end{aligned}
$$

Here we have introduced the notation $\Gamma_{(n)} \equiv \frac{1}{n !} \Gamma_{\mu_{1} \cdots \mu_{n}} \mathrm{~d} x^{\mu_{1}} \wedge \cdots \wedge \mathrm{d} x^{\mu_{n}}$, where $x^{\mu}$ are local coordinates. We also define

$$
\Omega \equiv J_{2}+\mathrm{i} J_{1}, \quad J \equiv J_{3}
$$

The canonical SU(2) structure is thus determined by $\left(K_{1}, K_{2}, J, \Omega\right)$. We note that $K_{1}$ and $K_{2}$ are orthonormal one-forms, and both are orthogonal to $J$ and $\Omega$, with $J \wedge \Omega=0$ and $2 J \wedge J=\Omega \wedge \bar{\Omega}$.

The $\mathrm{SU}(2)$ structure $\left(S, \vartheta, K_{1}, K_{2}, J, \Omega\right)$ that arises naturally from a supersymmetric solution is thus related to the canonical $\mathrm{SU}(2)$ structure by the square norm $S$ and angle $\vartheta$, via (2.8). For completeness we note that $\tilde{S}=-S \cos 2 \vartheta$.

Before proceeding, let us remark that the spinor $\epsilon$ is charged under the Abelian Rsymmetry gauge field $\mathcal{A}$, and thus it is rotated by a phase under gauge transformations. The two-form $\Omega$ is then rotated by the square of this phase. As a consequence we more precisely have a $\mathrm{U}(2)$ structure, as explained in [15]. Nevertheless, in this paper we will continue to refer to this as an $\mathrm{SU}(2)$ structure.

\subsection{Differential constraints}

We begin by introducing the one-form bilinear

$$
K \equiv \epsilon^{\dagger} \Gamma_{(1)} \epsilon=S \sin 2 \vartheta K_{1}
$$

Using the Killing spinor equation (2.3) and dilatino equation (2.4) one can show that $K$ is a Killing one-form, so that the dual vector field $\xi \equiv K^{\#}$ is a Killing vector. We may

\footnotetext{
${ }^{1}$ There are nevertheless interesting solutions for which $f \neq 0$. In particular the $1 / 2$ BPS solution constructed in [19] lies in this class.
} 
hence introduce a local coordinate $\psi$, so that $\xi=\partial_{\psi}$ and the metric is independent of $\psi$. From (2.11) it follows that we may write

$$
K_{1}=S \sin 2 \vartheta(\mathrm{d} \psi+\sigma),
$$

where $\mathcal{L}_{\xi} \sigma=0=i_{\xi} \sigma$. In fact, as shown in appendix $\mathrm{B}$, all of the supergravity fields and $\mathrm{SU}(2)$ structure are annihilated by $\mathcal{L}_{\xi}$, with the exception of the complex two-form $\Omega$. The spinor $\epsilon$ is a $\operatorname{spin}^{c}$ spinor, charged under the Abelian R-symmetry gauge field $\mathcal{A}$, and provided one makes the gauge choice (2.15) below then also $\mathcal{L}_{\xi} \Omega=0$. Thus the vector field $\xi=\partial_{\psi}$ generates a symmetry of the full solution.

The spinor equations (2.3), (2.4) impose further constraints on the supergravity fields and $\mathrm{SU}(2)$ structure. A more detailed analysis may be found in appendix B, while here we simply summarize the results. The $B$-field and R-symmetry gauge field strength $\mathcal{F}=\mathrm{d} \mathcal{A}$ may be written as

$$
\begin{aligned}
& B=\mathrm{i} K_{1} \wedge\left[\frac{3}{\sqrt{2} S \sin 2 \vartheta} \mathrm{d}(X S)+X^{-2} K_{2}\right]+B_{\perp}, \\
& \mathcal{F}=K_{1} \wedge \frac{\sqrt{2}}{S \sin 2 \vartheta} \mathrm{d}(X S \cos 2 \vartheta)+\mathcal{F}_{\perp},
\end{aligned}
$$

where $B_{\perp}$ and $\mathcal{F}_{\perp}$ have zero interior contraction with $\xi$. In particular (2.14) allows us to write

$$
\mathcal{A}=-\sqrt{2} X \cot 2 \vartheta K_{1}+\mathcal{A}_{\perp}
$$

where $i_{\xi} \mathcal{A}_{\perp}=0$ and we have made a partial gauge choice for $\mathcal{A}$. We note that

$$
\mathcal{F}_{\perp}=-\sqrt{2} X S \cos 2 \vartheta \mathrm{d} \sigma+\mathrm{d} \mathcal{A}_{\perp} .
$$

We may similarly write the component of $H=\mathrm{d} B$ perpendicular to $\xi$ as

$$
H_{\perp} \equiv \mathrm{i}\left[\frac{3}{\sqrt{2}} \mathrm{~d}(X S)+X^{-2} S \sin 2 \vartheta K_{2}\right] \wedge \mathrm{d} \sigma+\mathrm{d} B_{\perp} .
$$

Given these definitions, the spinor equations (2.3), (2.4) imply the following set of differential constraints on the $\mathrm{SU}(2)$ structure $\left(S, \vartheta, K_{1}, K_{2}, J, \Omega\right)$ :

$$
\begin{aligned}
X^{2} S^{2} \sin ^{2} 2 \vartheta \mathrm{d} \sigma= & \left.-\frac{2 \sqrt{2}}{3} X^{-1} S \cos 2 \vartheta J-\mathrm{i} X^{4} S \sin 2 \vartheta K_{1}\right\lrcorner * H_{\perp} \\
& +\sqrt{2} X S\left(\cos 2 \vartheta \mathcal{F}_{\perp}+\frac{2}{3} \mathrm{i} B_{\perp}\right) \\
\mathrm{d}\left(X^{-1} S \cos 2 \vartheta J\right)= & -\frac{3}{2 \sqrt{2}} \mathrm{~d}\left[(X S)^{2} \mathrm{~d} \sigma\right]+\mathrm{i} X S \mathrm{~d} B_{\perp} \\
& \left.+\frac{\sqrt{2}}{3} \mathrm{i} X^{-2} S \sin 2 \vartheta\left[K_{1}\right\lrcorner * B_{\perp}-K_{2} \wedge B_{\perp}\right], \\
\mathrm{d}\left(X^{-1} S J\right)= & -\sqrt{2} S \sin 2 \vartheta J \wedge K_{2}-\frac{3}{2 \sqrt{2}} \cos 2 \vartheta \mathrm{d}\left[(X S)^{2} \mathrm{~d} \sigma\right] \\
& \left.+\mathrm{i} X S \cos 2 \vartheta \mathrm{d} B_{\perp}-\frac{1}{\sqrt{2}} X^{-2} S \sin 2 \vartheta\left[K_{1}\right\lrcorner * \mathcal{F}_{\perp}-K_{2} \wedge \mathcal{F}_{\perp}\right],
\end{aligned}
$$




$$
\begin{aligned}
\mathrm{d}\left(S \sin 2 \vartheta J \wedge K_{2}\right)= & 0 \\
D_{\perp}\left(X^{-1} S \sin 2 \vartheta \Omega\right)= & -\sqrt{2} S \Omega \wedge K_{2} \\
S^{2} J \wedge \mathrm{d} \sigma= & \left.-\sqrt{2} S \cos 2 \vartheta\left(X+\frac{2}{3} X^{-3}\right) \frac{1}{2} J \wedge J+2 S K_{1}\right\lrcorner * \mathrm{~d} \vartheta \\
& +\frac{1}{\sqrt{2}} X^{-1} S J \wedge\left(\cos 2 \vartheta \mathrm{d} \mathcal{A}_{\perp}+\frac{2}{3} \mathrm{i} B_{\perp}\right) \\
S^{2} \Omega \wedge \mathrm{d} \sigma= & -2 \mathrm{i} S \mathrm{~d} \vartheta \wedge K_{2} \wedge \Omega+\frac{1}{\sqrt{2}} X^{-1} S \Omega \wedge\left(\cos 2 \vartheta \mathrm{d} \mathcal{A}_{\perp}+\frac{2}{3} \mathrm{i} B_{\perp}\right), \\
0= & \left.X^{4} K_{2}\right\lrcorner \mathrm{d}\left(X^{-3} S \sin 2 \vartheta\right)+\sqrt{2} S\left(X^{2}-\frac{2}{3} X^{-2}\right) \\
& \left.+\frac{1}{\sqrt{2}} S J\right\lrcorner\left(\mathcal{F}_{\perp}+\frac{2}{3} \mathrm{i} \cos 2 \vartheta B_{\perp}\right) .
\end{aligned}
$$

Here the covariant derivative is $D_{\perp}=\mathrm{d}+\mathrm{i} \mathcal{A}_{\perp} \wedge$, and the interior contraction of a $p$-form $\rho$ into a $q$-form $\lambda$ (with $q \geq p$ ) is the $(q-p)$-form $(\rho\lrcorner \lambda)_{\mu_{1} \cdots \mu_{q-p}} \equiv \frac{1}{p !} \rho^{\nu_{1} \cdots \nu_{p}} \lambda_{\nu_{1} \cdots \nu_{p} \mu_{1} \cdots \mu_{q-p}}$. Notice that the one-form $\sigma$ effectively determines $K_{1}$ via (2.12), while the supergravity fields enter the equations via $X, \mathcal{A}_{\perp}$ and $B_{\perp}$.

\subsection{Sufficiency}

In this section we shall argue that (2.18) are in fact equivalent to the original spinor equations (2.3), (2.4), and moreover as shown in appendix D these imply all but one component of the equations of motion (2.1), (2.2).

As in equation (2.7), we may decompose the Killing spinor as $\epsilon=\epsilon_{+}+\epsilon_{-}$, where $\epsilon_{ \pm}$ have definite chirality under $\Gamma_{7}$. Each of these defines an $\mathrm{SU}(3)$ structure in six dimensions, which is equivalent to specifying the real two-forms $\mathcal{J}_{ \pm} \equiv-\mathrm{i} \epsilon_{ \pm}^{\dagger} \Gamma_{(2)} \epsilon_{ \pm}$and complex threeforms $\Omega_{ \pm} \equiv \epsilon_{ \pm}^{\mathrm{T}} \Gamma_{(3)} \epsilon_{ \pm}$. For each choice of \pm , there exists a generalized connection with torsion $\nabla_{ \pm}^{(T)}$ which preserves the corresponding structure, i.e. $\nabla_{ \pm}^{(T)} \epsilon_{ \pm}=0$. One then defines the intrinsic torsion as $\tau_{ \pm} \equiv \nabla_{ \pm}^{(T)}-\nabla$, where $\nabla$ is the Levi-Civita connection. The exterior derivatives of $\mathcal{J}_{ \pm}$and $\Omega_{ \pm}$determine completely the corresponding intrinsic torsions. One can thus regard the Killing spinor equation as an equation that relates the exterior derivatives of $\mathcal{J}_{ \pm}$and $\Omega_{ \pm}$, on the left hand side of (2.3), to the supergravity fields on the right hand side. Since

$$
\begin{aligned}
\mathcal{J}_{ \pm} & =\frac{1}{2} S(1 \pm \cos 2 \vartheta)\left(J \mp K_{1} \wedge K_{2}\right), \\
\Omega_{ \pm} & =\frac{1}{2} S(1 \pm \cos 2 \vartheta) \Omega \wedge\left(\mp K_{1}+\mathrm{i} K_{2}\right),
\end{aligned}
$$

our equations (2.18) certainly contain this information, as they imply the exterior derivatives of all $k$-form bilinears, for $k \leq 3$ (this is clear from the analysis in appendix B). In fact they contain more than this information, as we have also used the dilatino constraint (2.4) to further simplify the equations.

It thus remains to show that (2.18) imply the dilatino equation (2.4). First we note that neither $\epsilon_{+}$nor $\epsilon_{-}$can be identically zero. For if $\epsilon_{ \pm}=0$, respectively, then we in fact have 
an $\mathrm{SU}(3)$ structure, rather than $\mathrm{SU}(2)$ structure, and the bilinear $W \equiv \epsilon^{\mathrm{T}} \Gamma_{(3)} \epsilon=\Omega_{\mp}$ is the corresponding complex three-form. However, since the left hand side of equation (B.8) of appendix B is identically zero, we would deduce that $\Omega_{\mp}=0$ and hence $\epsilon_{\mp}=0$. Thus on an open dense subset where $\epsilon_{ \pm}$are both non-zero, we have that $\left\{\epsilon_{ \pm}, \Gamma_{\mu} \epsilon_{ \pm}^{*}\right\}$ span the positive and negative chirality spin bundles $\mathcal{S}^{ \pm}$, respectively. In order for the dilatino equation to hold, it is therefore sufficient to check that the contraction of the right hand side of (2.4) with $\epsilon_{ \pm}^{\dagger}$ and $\epsilon_{ \pm}^{\mathrm{T}} \Gamma_{\mu}$ is zero. These are equivalent to two scalar and two one-form equations, respectively, that may be expressed in terms of bilinears. The corresponding equations may be found in appendix C. It is straightforward, but somewhat tedious, to show that these are indeed implied by (2.18).

We thus conclude that (2.18) are in fact necessary and sufficient for the original spinor equations (2.3), (2.4) to hold.

\subsection{Summary}

We have shown that a real supersymmetric solution to Euclidean Romans supergravity, with non-trivial Abelian R-symmetry gauge field $\mathcal{A}$, is described by an $\mathrm{SU}(2)$ structure $\left(S, \vartheta, K_{1}=S \sin 2 \vartheta(\mathrm{d} \psi+\sigma), K_{2}, J, \Omega\right)$ with corresponding metric

$$
\mathrm{d} s^{2}=S^{2} \sin ^{2} 2 \vartheta(\mathrm{d} \psi+\sigma)^{2}+K_{2}^{2}+g_{\mathrm{SU}(2)} .
$$

Here we may complete $K_{1}, K_{2}$ to an orthonormal frame $\left\{e^{a}, e^{5} \equiv K_{1}, e^{6} \equiv K_{2}\right\}, a=$ $1, \ldots, 4$, where

$$
g_{\mathrm{SU}(2)}=\sum_{a=1}^{4}\left(e^{a}\right)^{2}, \quad J=e^{1} \wedge e^{2}+e^{3} \wedge e^{4}, \quad \Omega=\left(e^{1}+\mathrm{i} e^{2}\right) \wedge\left(e^{3}+\mathrm{i} e^{4}\right) .
$$

The vector field $\xi=\partial_{\psi}$ is a Killing vector, and all supergravity fields and the $S U(2)$ structure are annihilated by $\mathcal{L}_{\xi}$ in the gauge for which

$$
\mathcal{A}=-\sqrt{2} X \cot 2 \vartheta K_{1}+\mathcal{A}_{\perp} .
$$

The Killing spinor equation (2.3) and dilatino equation (2.4) are then equivalent to imposing the differential constraints (2.18) on this structure, where $B_{\perp}$ is the component of the $B$-field with zero interior contraction with $\xi$. Moreover, these imply all of the equations of motion (2.1), (2.2) provided we also impose

$$
\begin{aligned}
0= & \left.\left.X^{4} S \sin 2 \vartheta \mathrm{d} \sigma \wedge\left(K_{1}\right\lrcorner * \mathrm{i} H_{\perp}\right)+\mathrm{d}\left[\frac{X^{4}}{S \sin 2 \vartheta} K_{1}\right\lrcorner * \mathrm{~d}\left(X^{-2} S \sin 2 \vartheta K_{2}\right)\right] \\
& \left.+\frac{2}{9} B_{\perp} \wedge B_{\perp}+\frac{1}{2} \mathcal{F}_{\perp} \wedge \mathcal{F}_{\perp}-\frac{4}{9} X^{-2} K_{1}\right\lrcorner *\left[\frac{3}{\sqrt{2} S \sin 2 \vartheta} \mathrm{d}(X S)+X^{-2} K_{2}\right] .
\end{aligned}
$$

This is the component of the $B$-field equation of motion in (2.1) that has zero interior contraction with $\xi$, where recall that $H_{\perp}$ is defined by (2.17). 


\section{Applications}

\subsection{Expansion at a conformal boundary}

In this section we determine the asymptotic form of the $\mathrm{SU}(2)$ structure at a conformal boundary. The aim is to make contact with the results of [15]. A similar holographic approach to constructing rigid supersymmetric backgrounds in lower dimensions was followed in $[22-24]$.

Given an asymptotically locally AdS solution we may introduce a radial coordinate $r$ with the conformal boundary located at $r=\infty$. The bosonic fields then admit an expansion of the form

$$
\begin{aligned}
\mathrm{d} s^{2} & =\frac{9}{2} \frac{\mathrm{d} r^{2}}{r^{2}}+r^{2}\left[g_{m n}^{(0)}+\frac{1}{r^{2}} g_{m n}^{(2)}+\cdots\right] \mathrm{d} x^{m} \mathrm{~d} x^{n}, \\
X & =1+\frac{1}{r^{2}} X_{2}+\cdots \\
B & =r b-\frac{1}{r^{2}} \mathrm{~d} r \wedge A^{(0)}+\cdots, \\
\mathcal{A} & =a+\cdots
\end{aligned}
$$

where recall $H=\mathrm{d} B$ and $\mathcal{F}=\mathrm{d} \mathcal{A}$. The five-dimensional coordinates on the conformal boundary are denoted $x^{m}$, with $m=1,2,3,4,5$. Some of the terms a priori present in these expansions are set to zero by the equations of motion.

In order to determine the corresponding expansion of the $\mathrm{SU}(2)$ structure, for this subsection we introduce the following explicit basis for $\operatorname{Cliff}(6,0)$ :

$$
\Gamma_{m}=\left(\begin{array}{cc}
0 & \mathrm{i} \gamma_{m} \\
-\mathrm{i} \gamma_{m} & 0
\end{array}\right), \quad \Gamma_{6}=\left(\begin{array}{cc}
0 & -1_{4} \\
-1_{4} & 0
\end{array}\right), \quad \Gamma_{7}=\left(\begin{array}{cc}
-1_{4} & 0 \\
0 & 1_{4}
\end{array}\right)
$$

where $\gamma_{m}$ are a Hermitian basis of $\operatorname{Cliff}(5,0)$. Notice that (3.2) is different to the basis used in the rest of the paper (where $\Gamma_{\mu}$ are purely imaginary), but instead coincides with the basis used in [15]. The asymptotic form of the metric implies the radial expansion of an orthonormal frame is

$$
E^{6}=-\frac{3}{\sqrt{2}} \frac{\mathrm{d} r}{r}, \quad E^{m}=r e^{m}+\cdots .
$$

The Killing spinor then has the following asymptotic expansion

$$
\epsilon=\sqrt{r}\left(\begin{array}{c}
\chi \\
-\mathrm{i} \chi
\end{array}\right)+\frac{1}{\sqrt{r}}\left(\begin{array}{c}
\varphi \\
\mathrm{i} \varphi
\end{array}\right)+\cdots
$$

From this, together with $S \equiv \epsilon^{\dagger} \epsilon$ and the definitions in (B.1), we deduce the following asymptotic expansion for the $\mathrm{SU}(2)$ structure:

$$
\begin{aligned}
& S=2 S^{(0)}(x) r+\cdots, \\
& \vartheta=\frac{\pi}{4}+\frac{\vartheta^{(0)}(x)}{r}+\cdots,
\end{aligned}
$$




$$
\begin{aligned}
K_{1} & =K_{1}^{(0)}(x) r+\cdots \\
K_{2} & =K_{2}^{(0)}(x)-\frac{3}{\sqrt{2}} \frac{\mathrm{d} r}{r}+\cdots, \\
J & =J^{(0)}(x) r^{2}+\cdots \\
\Omega & =\Omega^{(0)}(x) r^{2}+\cdots
\end{aligned}
$$

where the ellipses denote subleading terms. Inserting these expansions into (2.18) reduces to the following independent equations, at leading order in $r$ :

$$
\begin{aligned}
\mathrm{d} S^{(0)} & \left.=-\frac{\sqrt{2}}{3}\left(S^{(0)} K_{2}^{(0)}+\mathrm{i} S^{(0)} K_{1}^{(0)}\right\lrcorner b\right) \\
\mathrm{d}\left(S^{(0)} \vartheta^{(0)}\right) & \left.=-\frac{1}{2 \sqrt{2}} S^{(0)} K_{1}^{(0)}\right\lrcorner \mathrm{d} a \\
\mathrm{~d}\left(S^{(0)} K_{1}^{(0)}\right) & \left.=\frac{2 \sqrt{2}}{3}\left[2 \vartheta^{(0)} S^{(0)} J^{(0)}+S^{(0)} K_{1}^{(0)} \wedge K_{2}^{(0)}+\mathrm{i} S^{(0)} b-\frac{\mathrm{i}}{2} S^{(0)} K_{1}^{(0)}\right\lrcorner(* b)\right], \\
\mathrm{d}\left(S^{(0)} K_{2}^{(0)}\right) & \left.\left.=\mathrm{i} S^{(0)} K_{1}^{(0)}\right\lrcorner \mathrm{d} b-\mathrm{i} S^{(0)} K_{1}^{(0)}\right\lrcorner \mathrm{d}\left(\log S^{(0)}\right) b \\
\mathrm{~d}\left(S^{(0)} J^{(0)}\right) & =-\sqrt{2} K_{2}^{(0)} \wedge\left(S^{(0)} J^{(0)}\right) \\
\mathrm{d}\left(S^{(0)} \Omega^{(0)}\right) & =-\mathrm{i}\left(a-2 \sqrt{2} \vartheta^{(0)} K_{1}^{(0)}-\mathrm{i} \sqrt{2} K_{2}^{(0)}\right) \wedge\left(S^{(0)} \Omega^{(0)}\right)
\end{aligned}
$$

Here $*$ denotes the Hodge duality operator for the boundary metric $g^{(0)}$. We also note that the flux equation of motion (2.23) does not impose an independent constraint at leading order. The set of equations (3.6) is precisely the starting point for the purely field theory analysis of rigid supersymmetric five-manifold backgrounds carried out in [15].

\subsection{BPS Wilson loops}

The expectation value of Wilson loops in $\operatorname{USp}(2 N)$ SCFTs have been computed when the gauge theory is placed on the round five-sphere [25] or $\mathrm{SU}(3) \times \mathrm{U}(1)$ squashed fivesspheres [19]. Romans supergravity solutions dual to these backgrounds have also been constructed and successfully compared with the large $N$ gauge theory results. In this section we compute the regularised string action dual to the Wilson loops for any Romans solution with ball topology and $\mathrm{U}(1)^{3}$ symmetry, confirming one of the conjectures made by the authors in [19].

As shown in [19], the relevant string action is

$$
S_{\text {string }}=\int_{\Sigma_{2}} X^{-2} \operatorname{vol}_{2}+\mathrm{i} B-\frac{3}{\sqrt{2}} \operatorname{length}\left(\partial \Sigma_{2}\right),
$$

where the boundary counterterm regularizes the divergence arising from the infinite boundary length. We begin by writing

$$
B \equiv B_{1} \wedge K_{1}+B_{\perp}
$$


Comparing to (2.13) we see that

$$
X^{-2} K_{2}=-\frac{3}{\sqrt{2} S \sin 2 \vartheta} \mathrm{d}(X S)+\mathrm{i} B_{1} .
$$

It is natural to define the radial coordinate

$$
\rho \equiv X S
$$

Then

$$
X^{-2} K_{2}=-\frac{3 X}{\sqrt{2} \sin 2 \vartheta} \frac{\mathrm{d} \rho}{\rho}+\mathrm{i} B_{1} .
$$

Notice that in general $B_{1}$ has a component in the $\mathrm{d} \rho$ direction, and also $\mathrm{d} \rho$ is not orthogonal to $J$ and $\Omega$. However, we may still consider substituting (3.11) into the bilinears, at the expense of introducing the unknown $B_{1}$. From the point of view of asymptotically locally AdS solutions this is natural, since to leading order at large $\rho$ we see from (3.5) that $K_{2}$ is in the $\mathrm{d} \rho$ direction. Let us next wedge (3.11) with $K_{1}$. This reads

$$
X^{-2} K_{1} \wedge K_{2}+\mathrm{i}\left(B-B_{\perp}\right)=\frac{3}{\sqrt{2}} \mathrm{~d} \rho \wedge(\mathrm{d} \psi+\sigma) .
$$

The left hand side is precisely the (unregularized) action of a string wrapping the $K_{1}-K_{2}$ direction, while the right hand side is exact on the string worldsheet. In appendix E we show that such a string is supersymmetric. Notice that

$$
\left\|\partial_{\psi}\right\|=S \sin 2 \vartheta=\rho X^{-1} \sin 2 \vartheta=\rho+O(1 / \rho) .
$$

Here we have used the asymptotic expansions in section 3.1. Since the string wraps the $\partial_{\psi}$ direction, the boundary length is

$$
\operatorname{length}\left(\partial \Sigma_{2}\right)=\left\|\partial_{\psi}\right\| \int_{S^{1}} \mathrm{~d} \psi
$$

Integrating by parts the bulk action in (3.7), we see that the boundary counterterm simply cancels against the bulk contribution at infinity, leaving

$$
S_{\text {string }}=-\frac{3}{\sqrt{2}} \rho_{\text {origin }} \int_{S^{1}} \mathrm{~d} \psi
$$

where

$$
\rho_{\text {origin }}=\left.(X S)\right|_{\text {origin }}
$$

Here $\rho \in\left[\rho_{\text {origin }}, \infty\right)$. We next claim that for a solution with ball topology and $\mathrm{U}(1)^{3}$ isometry

$$
\left.(X S)\right|_{\text {origin }}=\frac{b_{1}+b_{2}+b_{3}}{\sqrt{2}}
$$


Here we write the supersymmetric Killing vector as

$$
\partial_{\psi}=\sum_{i=1}^{3} b_{i} \partial_{\varphi_{i}}
$$

where $\varphi_{i}, i=1,2,3$, have period $2 \pi$, and the orientations (and hence signs) will be fixed shortly. Combining (3.17) with (3.15) for a Wilson loop wrapping the $\varphi_{i}$ circle we obtain

$$
S_{\text {string }}=-9 \pi \frac{b_{1}+b_{2}+b_{3}}{3 b_{i}},
$$

where $\int_{S^{1}} \mathrm{~d} \psi=2 \pi / b_{i}$. This is precisely the Wilson loop conjecture made by the authors in [19].

Thus it remains to prove (3.17). Geometrically, the $b_{i}$ arise as the skew eigenvalues of the two-form $\mathrm{d} K$ at the origin (recall that $K=S \sin 2 \vartheta K_{1}$ is a Killing one-form). That is, raising an index of $\mathrm{d} K$ to obtain a skew-symmetric $6 \times 6$ matrix in an orthonormal frame, at the origin we have

$$
\left.(\mathrm{d} K)\right|_{\text {origin }}=\left(\begin{array}{ccc}
R_{1} & 0 & 0 \\
0 & R_{2} & 0 \\
0 & 0 & R_{3}
\end{array}\right), \quad R_{i}=\left(\begin{array}{cc}
0 & -b_{i} \\
b_{i} & 0
\end{array}\right) .
$$

This follows from a simple local calculation. Specifically, at the origin we may introduce three sets of polar coordinates $\left(\rho_{i}, \varphi_{i}\right), i=1,2,3$, and write the leading order flat metric as

$$
\mathrm{d} s_{\text {flat }}^{2}=\sum_{i=1}^{3} \mathrm{~d} \rho_{i}^{2}+\rho_{i}^{2} \mathrm{~d} \varphi_{i}^{2} .
$$

One can then compute $\mathrm{d} K$ at the origin using this local metric, where $K=\sum_{i=1}^{3} b_{i} \rho_{i}^{2} \mathrm{~d} \varphi_{i}$ is the dual one-form to $\partial_{\psi}$. In the orthonormal frame

$$
e_{2 i-1}=\mathrm{d} \rho_{i}, \quad e_{2 i}=\rho_{i} \mathrm{~d} \varphi_{i}, \quad i=1,2,3,
$$

at the origin this gives precisely (3.20). Our solution is also equipped with a six-dimensional almost complex structure, which as a two-form reads

$$
\mathcal{J}=K_{1} \wedge K_{2}+J
$$

In the same frame this reads

$$
\mathcal{J}=\left(\begin{array}{ccc}
\varepsilon & 0 & 0 \\
0 & \varepsilon & 0 \\
0 & 0 & \varepsilon
\end{array}\right), \quad \varepsilon=\left(\begin{array}{cc}
0 & -1 \\
1 & 0
\end{array}\right)
$$

Thus $\mathcal{J}\left(e_{1}\right)=e_{2}$, etc. Notice this fixes the orientations of the $\varphi_{i}$. Then

$$
\mathcal{J}\lrcorner\left.\mathrm{d} K\right|_{\text {origin }}=2\left(b_{1}+b_{2}+b_{3}\right) .
$$


Let us now look at computing the same quantity using the bilinear equations. We have

$$
\left.K_{1}\right\lrcorner \mathrm{d} K=\frac{1}{X^{2}}\left[-\frac{1}{S \sin 2 \vartheta} \mathrm{d}\left(X^{2} S^{2} \sin ^{2} 2 \vartheta\right)+2 X S \sin 2 \vartheta \mathrm{d} X\right] .
$$

$K$ has norm $S \sin 2 \vartheta$, which by definition is zero at the origin. Contracting $K_{2}$ into (3.26) and restricting to the origin we hence find

$$
\left.\left.\left(K_{1} \wedge K_{2}\right)\right\lrcorner\left.\mathrm{d} K\right|_{\text {origin }}=-2 K_{2}\right\lrcorner\left.\mathrm{d}(S \sin 2 \vartheta)\right|_{\text {origin }},
$$

where we have assumed that $X$ is regular at the origin (and we shall make similar regularity assumptions for other fields in what follows). We next compute

$$
\left.J\lrcorner \mathrm{~d} K=(S \sin 2 \vartheta)^{2} J\right\lrcorner \mathrm{d} \sigma,
$$

which thus tends to zero at the origin. Finally contracting $K_{2}$ into (B.24), and restricting to the origin, we find

$$
\left.K_{2}\right\lrcorner\left.\mathrm{d}(S \sin 2 \vartheta)\right|_{\text {origin }}=-\left.\sqrt{2}(X S)\right|_{\text {origin }} .
$$

Combined with (3.27), this shows that

$$
\mathcal{J}\lrcorner\left.\mathrm{d} K\right|_{\text {origin }}=\left.2 \sqrt{2}(X S)\right|_{\text {origin }},
$$

which together with (3.25) proves (3.17).

\subsection{Squashed Sasaki-Einstein solutions}

The system of equations for the $\mathrm{SU}(2)$ structure in section 2 is too complicated to solve in general; to find solutions one needs to make some additional assumptions. In this section we consider an ansatz that naturally generalizes the $1 / 4$ BPS solutions (and their 1/2 BPS limit) found in [19].

We begin by making the following ansatz for the supergravity fields ${ }^{2}$

$$
\begin{aligned}
\mathrm{d} s^{2} & =\alpha^{2}(r) \mathrm{d} r^{2}+\gamma^{2}(r)(\mathrm{d} \psi+\sigma)^{2}+\beta^{2}(r) \mathrm{d} s_{\mathrm{KE}}^{2}, \\
B & =p(r) \mathrm{d} r \wedge(\mathrm{d} \psi+\sigma)+\frac{1}{2} q(r) \mathrm{d} \sigma \\
\mathcal{A} & =f(r)(\mathrm{d} \psi+\sigma)-3 \mathrm{~d} \psi \\
X & =X(r) .
\end{aligned}
$$

Here we take $\mathrm{d} s_{\mathrm{KE}}^{2}$ to be a four-dimensional positively curved Kähler-Einstein metric, so that a constant $r$ hypersurface is a squashed Sasaki-Einstein five-manifold. Concretely, this means that $\mathrm{d} \psi+\sigma$ is a global contact one-form on such a hypersurface, with

$$
\mathrm{d} \sigma=2 \omega_{\mathrm{KE}} .
$$

\footnotetext{
${ }^{2}$ Recall that the formula $(2.22)$ for the gauge field $\mathcal{A}$ requires a specific gauge choice. However, in [19] this was presented in a different gauge. This accounts for the factor of $-3 \mathrm{~d} \psi$ in (3.31).
} 
The ansatz (3.31) reduces to that in [19] on taking the Kähler-Einstein metric to be the Fubini-Study metric on $\mathbb{C P}^{2}$. Notice also that in writing (3.31) we have taken the supersymmetric Killing vector $\partial_{\psi}$ to coincide with the Reeb vector field of the squashed Sasaki-Einstein manifold.

Comparing to section 2, and identifying the four-dimensional SU(2) structure metric in $(2.20)$ with $\beta^{2}(r) \mathrm{d} s_{\mathrm{KE}}^{2}$, allows us to identify

$$
S \sin 2 \vartheta=\gamma(r), \quad K_{2}=-\alpha(r) \mathrm{d} r, \quad \Omega=\beta^{2}(r) \Omega_{\mathrm{KE}}, \quad J=-\beta^{2}(r) \omega_{\mathrm{KE}},
$$

where $\Omega_{\mathrm{KE}}$ satisfies $^{3}$

$$
\mathrm{d} \Omega_{\mathrm{KE}}=-3 \mathrm{i} \sigma \wedge \Omega_{\mathrm{KE}} .
$$

We take $S=S(r), \vartheta=\vartheta(r)$. From the remaning supergravity fields, we similarly read off

$$
f(r)=3-\sqrt{2} X S \cos 2 \vartheta, \quad \mathcal{F}_{\perp}=2 f(r) \omega_{\mathrm{KE}}, \quad B_{\perp}=q(r) \omega_{\mathrm{KE}} .
$$

Substituting these into the differential constraints (2.18) and flux equation of motion (2.23) then reduces to the following independent ODEs:

$$
\begin{aligned}
& 0=\mathrm{i} X^{3}\left(2 p-q^{\prime}\right) \sin 2 \vartheta+\frac{2 \sqrt{2}}{3} \alpha\left[\mathrm{i} q+\left(9+\beta^{2} X^{-2}\right) \cos 2 \vartheta\right]-\alpha X S(3+\cos 4 \vartheta), \\
& 0=\frac{\mathrm{d}}{\mathrm{d} r}\left(X^{-1} S \beta^{2} \cos 2 \vartheta\right)-3 \sqrt{2} X S \frac{\mathrm{d}}{\mathrm{d} r}(X S)+\mathrm{i} X S q^{\prime}, \\
& 0=\frac{\mathrm{d}}{\mathrm{d} r}\left(X^{-1} S \beta^{2} \sin 2 \vartheta\right)-\sqrt{2} S \alpha \beta^{2}, \\
& 0=-2 X S+3 \sqrt{2} \cos 2 \vartheta+\mathrm{i} \frac{\sqrt{2}}{3} q+\frac{1}{\sqrt{2}}\left(\frac{2}{3} X^{-2}+X^{2}\right) \beta^{2} \cos 2 \vartheta-\beta^{2} X \alpha^{-1} \vartheta^{\prime}, \\
& 0=-\sqrt{2} \alpha S\left[\left(3 X^{4}+1\right) \beta^{2}+18 X^{2}\right] \sin 2 \vartheta+X^{2}\left(12 X S \beta^{\prime}+\sqrt{2} \mathrm{i} p \beta\right) \beta, \\
& 0=-\frac{p \beta^{4} \csc 2 \vartheta}{\alpha X^{2} S}+6 \sqrt{2} q X S-\mathrm{i} q^{2}-\frac{6 \sqrt{2} \mathrm{i} S \beta^{2} \cos 2 \vartheta}{X}+18 \mathrm{i} X^{2} S^{2}-81 \mathrm{i} .
\end{aligned}
$$

Notice that as a consequence of parametrization invariance one is free to specify the function $\beta=\beta(r)$. Hence (3.36) are six coupled ODEs for the six functions $(X, S, \vartheta, \alpha, p, q)$. Furthermore, notice that they are independent of the choice of Kähler-Einstein metric, and are thus equivalent to the equations studied in [19]. In the latter reference we constructed a two-parameter family of $1 / 4$ BPS solutions, as a series expansion both around the conformal boundary at $r=\infty$, and as an expansion around Euclidean AdS. Specifically, the parameters are

$$
\left.f_{0} \equiv f(r)\right|_{\text {boundary }},\left.\quad s^{-1} \equiv \frac{\gamma(r)}{\beta(r)}\right|_{\text {boundary }} .
$$

We hence automatically construct new solutions, with an arbitrary squashed SasakiEinstein five-manifold, with squashing parameter $s$, as conformal boundary. Setting

\footnotetext{
${ }^{3}$ We have chosen sign conventions so as to agree with those of [19].
} 
$s=1$ and $f_{0}=0$, the conformal boundary is a Sasaki-Einstein manifold with metric $\mathrm{d} s_{\mathrm{SE}}^{2}=(\mathrm{d} \psi+\sigma)^{2}+\mathrm{d} s_{\mathrm{KE}}^{2}$, and in the bulk the only non-trivial field is the metric, which is a "hyperbolic cone"

$$
\mathrm{d} s_{6}^{2}=\frac{\mathrm{d} r^{2}}{1+\frac{2}{9} r^{2}}+r^{2} \mathrm{~d} s_{\mathrm{SE}}^{2}
$$

When $\mathrm{d} s_{\mathrm{SE}}^{2}$ is the round five-sphere this is simply Euclidean $\mathrm{AdS}_{6}$, while more generally (3.38) has an isolated Calabi-Yau cone singularity at $r=0$. The solutions with general $s$ and $f_{0}$ have the same behaviour near the tip of the cone/origin, and thus in general these supergravity solutions have a Calabi-Yau singularity. Nevertheless, this singularity does not lead to any UV divergences in the holographic free energy or Wilson loop VEVs. Although we were unable to solve the system (3.36) analytically, see the end of section 3.4 for further discussion.

Any solution to Romans $F(4)$ supergravity uplifts to a solution of massive type IIA supergravity, as a warped product $M_{6} \times S^{4}$ [27]. For an asymptotically locally AdS solution $M_{6}$, these are expected to be the gravity duals to a certain family of $\operatorname{USp}(2 N)$ gauge theories, defined on the conformal boundary of $M_{6}$. The gauge theories arise from a system of $N$ D4-branes, $N_{f}$ of D8-branes and an orientifold plane. This data is captured in the six-dimensional effective Newton constant [26]

$$
G_{N}=\frac{15 \pi \sqrt{8-N_{f}}}{4 \sqrt{2} N^{5 / 2}} .
$$

Recall that the two-parameter family of solutions constructed in this section reduce to the 1/4 BPS family in [19] when the Kähler-Einstein metric is taken to be the Fubini-Study metric on $\mathbb{C P}^{2}$. The computation of the holographic free energy then very closely follows that in [19]. The upshot is that

$$
\mathcal{F}_{\text {gravity }}=I_{\text {renormalized }}=-\frac{27}{4 \pi G_{N}} \cdot \operatorname{vol}(\mathrm{SE}),
$$

is independent of the two parameters $s$ and $f_{0}$. Notice that the volume vol(SE) appearing in (3.40) is that of the Sasaki-Einstein metric, which is the conformal boundary metric when $s=1$, even though (3.40) holds for all $s$.

Comparison to field theory. We would like to compare (3.40) with the corresponding large $N$ field theory calculation. This involves computing the localized partition function of the $\operatorname{USp}(2 N)$ gauge theories on a squashed Sasaki-Einstein background, and taking the $N \rightarrow \infty$ limit. In [28] the perturbative partition function of an arbitrary $\mathcal{N}=1$ supersymmetric gauge theory was computed on a general $\mathrm{U}(1)^{3}$-invariant Sasaki-Einstein five-manifold. For a gauge theory with gauge group $G$ and a matter hypermultiplet in an arbitrary representation $\mathcal{R}$, the localized perturbative partition function is

$$
Z_{\text {pert }}^{\mathrm{SE}}=\int_{\mathrm{t}} \mathrm{d} a \mathrm{e}^{-S_{\mathrm{cl}}} \frac{\prod_{\alpha} S_{3}^{\mathrm{SE}}[\mathrm{i} \alpha(a) ; \vec{\xi}]}{\prod_{\rho} S_{3}^{\mathrm{SE}}\left[\mathrm{i} \rho(a)+\frac{3}{2} ; \vec{\xi}\right]} .
$$


The integration in $a$ is over the Cartan $\mathfrak{t}$ of the gauge group. The products are over roots $\alpha$ of $G$ and weights $\rho$ of the representation $\mathcal{R}$, and we have denoted by $S_{\mathrm{cl}}$ the classical action evaluated on the localization locus. Furthermore $S_{3}^{\mathrm{SE}}[x ; \vec{\xi}]$ is a generalized version of the triple-sine function

$$
S_{3}^{\mathrm{SE}}[x ; \vec{\xi}] \equiv \prod_{\vec{m}}(\vec{m} \cdot \vec{\xi}+x)(\vec{m} \cdot \vec{\xi}+\vec{\xi} \cdot \vec{\xi}-x) .
$$

Here $\vec{m}=\left(m_{1}, m_{2}, m_{3}\right)$ runs over the charge lattice of holomorphic functions on the CalabiYau cone over the Sasaki-Einstein five-manifold, where $m_{i}$ is the charge under the $i$ th $\mathrm{U}(1)$ symmetry. Furthermore, we have written the supersymmetric (Reeb) vector field as

$$
\xi=\sum_{i=1}^{3} \xi_{i} \partial_{\varphi_{i}}
$$

where $\vec{\xi}=\left(\xi_{1}, \xi_{2}, \xi_{3}\right)$ and $\partial_{\varphi_{i}}$ generate the $\mathrm{U}(1)^{3}$ isometry. For example, for the round $S^{5}$ the Calabi-Yau cone is simply $\mathbb{C}^{3}$, with a basis of holomorphic functions $z_{1}^{m_{1}} z_{2}^{m_{2}} z_{3}^{m_{3}}$, where $m_{i} \in \mathbb{Z}_{\geq 0}$. In this case, (3.42) reduces to the standard triple-sine function.

We are interested in evaluating (3.41) for the $\operatorname{USp}(2 N)$ gauge theories, in the large $N$ limit. This involves the asymptotics of the hypermultiplet and vectormultiplet contributions computed in [28]:

$$
\begin{aligned}
\log S_{3}^{\mathrm{SE}}[x ; \vec{\xi}] & \sim-\mathrm{i} \pi \operatorname{sgn}(\operatorname{Im} x)\left[\left(\frac{x^{3}}{6}+\frac{3 x}{4}\right) \frac{\operatorname{vol}(\mathrm{SE})}{\pi^{3}}+\frac{x}{24 \pi} \sum_{I} \beta_{I}\right], \\
\log S_{3}^{\mathrm{SE}}\left[x+\frac{3}{2} ; \vec{\xi}\right] & \sim \mathrm{i} \pi \operatorname{sgn}(\operatorname{Im} x)\left[\left(\frac{x^{3}}{6}-\frac{3 x}{8}\right) \frac{\operatorname{vol}(\mathrm{SE})}{\pi^{3}}+\frac{x}{24 \pi} \sum_{I} \beta_{I}\right] .
\end{aligned}
$$

Here $\beta_{I}$ are certain parameters defined in [28], which will not enter the final result. ${ }^{4}$ We may then compute the leading contribution to the partition function at large $N$ using a saddle point method. One specifies an element of the Cartan subalgebra of $\operatorname{USp}(2 N)$ by its eigenvalues $\left\{\lambda_{1}, \ldots, \lambda_{N}\right\}$. In the large $N$ saddle point these behave as $\lambda_{n} \sim N^{1 / 2} x_{n}$. One then introduces an eigenvalue density

$$
\rho(x)=\frac{1}{N} \sum_{n} \delta\left(x-x_{n}\right),
$$

which has support on a finite interval $\left[0, x_{\star}\right]$. Solving the saddle point approximation to the above matrix model, we find

$$
\rho(x)=\frac{4\left(8-N_{f}\right) x}{9}, \quad \text { and } \quad x_{\star}=\frac{3}{\sqrt{2} \sqrt{8-N_{f}}},
$$

which leads to the final result for the large $N$ free energy

$$
\mathcal{F}_{\text {gauge theory }}=-\frac{9 \sqrt{2}}{5 \pi^{2} \sqrt{8-N_{f}}} \operatorname{vol}(\mathrm{SE}) N^{5 / 2}+o\left(N^{5 / 2}\right) .
$$

This precisely agrees with (3.40).

\footnotetext{
${ }^{4} \beta_{I}$ is the length of the $I$ th closed Reeb orbit.
} 
The field theory computation above is for the Sasaki-Einstein conformal boundary, with $s=1$ and $f_{0}=0$. On the other hand, in [15] we conjectured that the partition function should depend only on the holomorphic foliation generated by the Killing vector $\xi$. Since this is independent of $s$ and $f_{0}$, this conjecture implies that (3.47) holds for the entire two-parameter family of $1 / 4$ BPS backgrounds. Since (3.47) agrees with (3.40), this lends credence to the conjecture. We also regard this as evidence that the $1 / 4$ BPS family of supergravity backgrounds is the correct holographic dual, in spite of the Calabi-Yau singularity at the origin.

BPS Wilson loops. Finally, let us discuss the computation of the VEV of BPS Wilson loops on both sides of the correspondence. Following a similar computation to that in [19], in the large $N$ matrix model for the gauge theory this is given by

$$
\langle W\rangle=\int_{0}^{x_{\star}} \mathrm{e}^{\lambda(x) \beta_{I}} \rho(x) \mathrm{d} x,
$$

where $\beta_{I}$ is the length of the closed Reeb orbit wrapped by the Wilson loop. ${ }^{5}$ At large $N$ one hence obtains

$$
\log \langle W\rangle=x_{\star} \beta_{I} N^{1 / 2}+o\left(N^{1 / 2}\right)
$$

On the other hand, in the dual supergravity solution this corresponds to a fundamental string wrapping the circle of length $\beta_{I}$, together with the radial direction $r$. We find that the regularized action is

$$
S_{\text {string }}=-\frac{3}{\sqrt{2} \sqrt{8-N_{f}}} \beta_{I} N^{1 / 2} .
$$

This should be identified with $-\log \langle W\rangle$ in field theory, and we find perfect agreement.

\subsection{Analytic 3/4 BPS solution}

In this section we give some details of a new analytic supersymmetric solution to Euclidean six-dimensional Romans supergravity. This corresponds to the 3/4 BPS squashed sphere, constructed as a perturbation expansion in [19]. As shown in [19] an interesting family of solutions arises by considering the following $\mathrm{SU}(3) \times \mathrm{U}(1)$ symmetric ansatz for the supergravity fields

$$
\begin{aligned}
\mathrm{d} s_{6}^{2}= & \alpha^{2}(r) \mathrm{d} r^{2}+\gamma^{2}(r)(\mathrm{d} \tau+C)^{2}+\beta^{2}(r)\left[\mathrm{d} \sigma^{2}+\frac{1}{4} \sin ^{2} \sigma\left(\mathrm{d} \theta^{2}+\sin ^{2} \theta \mathrm{d} \varphi^{2}\right)\right. \\
& \left.+\frac{1}{4} \cos ^{2} \sigma \sin ^{2} \sigma(\mathrm{d} \beta+\cos \theta \mathrm{d} \varphi)^{2}\right], \\
B & =p(r) \mathrm{d} r \wedge(\mathrm{d} \tau+C)+\frac{1}{2} q(r) \mathrm{d} C, \\
A^{i} & =f^{i}(r)(\mathrm{d} \tau+C),
\end{aligned}
$$

\footnotetext{
${ }^{5}$ Recall that the computation of [28] is valid for a $\mathrm{U}(1)^{3}$-invariant Sasaki-Einstein manifold, for which the index $I$ runs over the rays of the corresponding polyhedral cone.
} 
where

$$
C \equiv-\frac{1}{2} \sin ^{2} \sigma(\mathrm{d} \beta+\cos \theta \mathrm{d} \varphi),
$$

together with $X=X(r)$. The equations of motion for the background $\mathrm{SU}(2)_{R}$ gauge field imply

$$
f^{i}(r)=\kappa_{i} f(r) .
$$

The equations for the other fields then depend only on the $\mathrm{SU}(2) \sim \mathrm{SO}(3)$ invariant $\kappa_{1}^{2}+\kappa_{2}^{2}+\kappa_{3}^{2}$, which we can set to one by rescaling $f(r)$. The set of equations for the fields involved in the ansatz have been listed in the appendix B to [19]. In addition, if the solution is supersymmetric there exists a Killing spinor. For the case of the $3 / 4$ BPS solution the Killing spinor depends on four extra functions, denoted $k_{i}(r), i=1,2,3,4$ in [19], which, together with the fields above, satisfy first order constraints as a result of supersymmetry. Although, as shown in this paper, these constraints are equivalent to the original equations of motions (upon supplementing them with one extra second order equation), we found them more convenient in order to find an analytic form for the solution.

The solution depends on a single parameter $s$, the squashing parameter, but it is convenient to parametrize it in terms of $b_{1}=1+\sqrt{1-s^{2}}$ and $b_{2}=1-\sqrt{1-s^{2}}$, introduced in [19]. The high amount of supersymmetry implies a large number of constraints (many of them algebraic) which can be used to eliminate all the fields in favour of $k_{2}(r), k_{3}(r), X(r)$ and $\beta(r)$. For instance

$$
\begin{aligned}
k_{1}(r) & =b_{2}\left(b_{1}+b_{2}\right) \frac{k_{2}(r) \beta(r)}{b_{2} k_{2}^{2}(r)+b_{1} k_{3}^{2}(r)}, \\
k_{4}(r) & =b_{1}\left(b_{1}+b_{2}\right) \frac{k_{3}(r) \beta(r)}{b_{2} k_{2}^{2}(r)+b_{1} k_{3}^{2}(r)}, \\
\gamma(r) & =\left(b_{1}+b_{2}\right) \frac{k_{2}(r) k_{3}(r) \beta(r)}{b_{2} k_{2}^{2}(r)+b_{1} k_{3}^{2}(r)},
\end{aligned}
$$

while the expressions for the remaining fields are more complicated. As a consequence of reparametrization invariance we can demand that $k_{2}, k_{3}$ and $X$ depend on $r$ only through $\beta(r)$. It is then convenient to introduce a new variable $\zeta$ :

$$
\left(b_{1}+b_{2}\right) \sqrt{b_{1} b_{2}} \beta(r) \equiv \zeta .
$$

The remaining equations can be used to eliminate further fields and we end up with a single equation for $v(\zeta) \equiv \zeta^{2} X^{2}(\zeta)$ :

$$
v^{\prime}(\zeta)=4 \zeta^{3} \frac{\left(b_{1}+b_{2}\right)^{2}\left(b_{1}+2 b_{2}\right)^{2}+2 v(\zeta)}{\zeta^{4}+3\left(b_{1}+b_{2}\right)^{3}\left(b_{1}+2 b_{2}\right) v(\zeta)+3 v^{2}(\zeta)},
$$

which can be simply solved, for instance, with Mathematica. Equation (3.56) has two inequivalent solutions, each of them depending on a constant of integration. Of those only one has the correct boundary condition at infinity $v(\zeta)=\zeta^{2}+\cdots$. The constant of integration can then be fixed by requiring regularity at the origin for $X(\zeta)$, which implies 
$v(0)=0$. This fixes the solution uniquely. Although the explicit solution is too cumbersome to be written here, we give the expansion of $X(\zeta)$ for small and large values of $\zeta$ :

$$
\begin{array}{rlrl}
X(\zeta)= & \left(\frac{2\left(b_{1}+2 b_{2}\right)}{3\left(b_{1}+b_{2}\right)}\right)^{1 / 4}+\cdots, & & \zeta \ll 1, \\
X(\zeta)= & 1-\frac{\left(b_{1}-b_{2}\right)\left(b_{1}+b_{2}\right)^{2}\left(b_{1}+2 b_{2}\right)}{4} \frac{1}{\zeta^{2}} & \\
& +\frac{\left(b_{1}-b_{2}\right)\left(b_{1}+b_{2}\right)^{3}\left(b_{1}+2 b_{2}\right)^{2}}{2 \sqrt{2}} \frac{1}{\zeta^{3}}+\cdots, & \zeta \gg 1 .
\end{array}
$$

For instance, these expansions allow us to fix the parameter $\kappa$ introduced in [19]. We obtain

$$
\kappa=\frac{2 \sqrt{2}\left(3-\sqrt{1-s^{2}}\right)^{2}\left(1-s^{2}+\sqrt{1-s^{2}}\right)}{27 \sqrt{3} s^{5}} .
$$

Finally, let us remark that although cumbersome, the solution contains only roots and rational functions.

Comments on the $\mathbf{1 / 4}$ and $\mathbf{1 / 2}$ BPS solutions. The $1 / 4$ BPS squashed sphere solution considered in [19] is much harder to obtain, the reason being the smaller degree of supersymmetry. More precisely, the Killing spinor now depends on only two new functions $k_{1}(r)$ and $k_{2}(r)$, but the number of constraints is much smaller. A related issue is that now there are no natural "constants of motion" such as $b_{1}$ and $b_{2}$ to parametrize the solution with. Proceeding as before one can write two (third order and very cumbersome!) equations for two of the fields, for instance $X(\zeta)$ and $f(\zeta)$. After requiring regularity at the origin this should lead to a two-parameter family of solutions ( $s$ and $f_{0}$ introduced in (3.37)). These equations, however, are very complicated and we haven't managed to solve them exactly. Before proceeding, two comments are in order: first, these two equations can be solved in different limits, and reproduce the $1 / 4$ BPS solution in the limits studied in [19]. Furthermore, in order to obtain these two equations it is necessary to supplement the bilinear equations with (2.23). Otherwise, we would obtain only one equation for two fields. This example shows that the differential constraints (2.18) do indeed need to be supplemented by equation (2.23).

We can also consider the special case $f(\zeta)=0$. In this case the $1 / 4$ BPS solution reduces to the $1 / 2$ BPS solution studied in [19]. Although not covered by our analysis in this paper because the bilinear $\epsilon^{\mathrm{T}} \epsilon \neq 0$, the $1 / 2$ BPS solution is a limit of the $1 / 4$ BPS solution, where one of the two parameters, namely $f_{0}$, vanishes. The final equation for $X(\zeta)$, with $\beta(r) \equiv \zeta$ is still rather involved, but it can be solved analytically in an interesting limit. Denoting $X(0)=x_{0}$ one can explicitly check the solution takes the following form

$$
v(\zeta)=v_{0}\left(x_{0} \zeta\right)+\frac{1}{x_{0}^{4}} v_{1}\left(x_{0} \zeta\right)+\cdots,
$$

where recall $v(\zeta) \equiv \zeta^{2} X^{2}(\zeta)$ and $v_{0}(y)$ satisfies a simple equation

$$
v_{0}^{\prime \prime}(y)=3 \frac{v_{0}^{\prime}(y)}{y}-\frac{\left(6+v_{0}(y)\right) v_{0}^{\prime}(y)^{2}}{6 v_{0}(y)},
$$


whose solution with correct boundary conditions is

$$
v_{0}(y)=1+\mathcal{W}\left(\frac{y^{4}-72 \mathrm{e}}{72 \mathrm{e}}\right) .
$$

Here $\mathcal{W}(z)$ is the Lambert $\mathrm{W}$ function or product logarithm, namely $\mathcal{W}(z) \mathrm{e}^{\mathcal{W}(z)}=z$. Hence, as opposed to the $3 / 4$ BPS solution, this solution contains special functions.

\section{Discussion}

In this paper we have presented a systematic study of supersymmetric solutions to sixdimensional Euclidean Romans supergravity. These are characterized by an SU(2) structure. We then used these results to study a number of different applications.

Our results raise a number of interesting questions and directions for future work. Firstly, the gravity duals to (squashed) Sasaki-Einstein backgrounds we constructed have isolated Calabi-Yau singularities. However, as we have seen, the singularity does not contribute additional (UV) divergences to the free energy and Wilson loop, and moreover the supergravity computations agree with the gauge theory results. It is thus natural to conjecture that these are the correct gravity duals. More precisely, although one expects some stringy degrees of freedom to be supported at the singularity, we expect that these should not contribute to leading order at large $N$. Notice in any case that the uplift to massive IIA is also singular (along the internal $S^{4}$ ), even for Euclidean $\operatorname{AdS}_{6}[29,30]$.

Using the technology developed in the paper, we have computed the VEV of the holographic dual of a supersymmetric Wilson loop for a general class of solutions, thus proving one of the conjectures of [19]. Another conjecture made in that paper makes a specific prediction for the holographic free energy for the same class of backgrounds. It would be interesting to prove this conjecture. Note that this computation is more involved than that for the Wilson loop; in particular the structure of the counterterms is much more complicated.

Finally, it would be interesting to construct further analytic solutions, including solutions with different topology.

\section{Acknowledgments}

The work of L. F. A., M. F. and P. R. is supported by ERC STG grant 306260. L. F. A. is a Wolfson Royal Society Research Merit Award holder. C. M. G. is supported by a CNPq scholarship. J. F. S. is supported by the Royal Society.

\section{A Useful identities}

From the dilatino equation (2.4) one can derive the following useful identities

$$
\begin{aligned}
\left(\partial^{\mu} X\right) \epsilon^{\dagger}\left[\mathbb{A}, \Gamma_{\mu}\right]_{\mp} \epsilon= & -\frac{\mathrm{i}}{2 \sqrt{2}}\left(X^{2}-X^{-2}\right) \epsilon^{\dagger}\left[\mathbb{A}, \Gamma_{7}\right]_{ \pm} \epsilon+\frac{1}{24} X^{3} H^{\mu \nu \rho} \epsilon^{\dagger}\left[\mathbb{A}, \Gamma_{\mu \nu \rho} \Gamma_{7}\right]_{ \pm} \epsilon \\
& +\frac{\mathrm{i}}{12 \sqrt{2}} B^{\mu \nu} \epsilon^{\dagger}\left[\mathbb{A}, \Gamma_{\mu \nu}\right]_{ \pm} \epsilon-\frac{1}{8 \sqrt{2}} \mathcal{F}^{\mu \nu} \epsilon^{\dagger}\left[\mathbb{A}, \Gamma_{\mu \nu} \Gamma_{7}\right]_{ \pm} \epsilon,
\end{aligned}
$$




$$
\begin{aligned}
\left(\partial^{\mu} X\right) \epsilon^{\mathrm{T}}\left[\mathbb{A}, \Gamma_{\mu}\right]_{\mp} \epsilon= & -\frac{\mathrm{i}}{2 \sqrt{2}}\left(X^{2}-X^{-2}\right) \epsilon^{\mathrm{T}}\left[\mathbb{A}, \Gamma_{7}\right]_{\mp} \epsilon+\frac{1}{24} X^{3} H^{\mu \nu \rho} \epsilon^{\mathrm{T}}\left[\mathbb{A}, \Gamma_{\mu \nu \rho} \Gamma_{7}\right]_{ \pm} \epsilon \\
& +\frac{\mathrm{i}}{12 \sqrt{2}} B^{\mu \nu} \epsilon^{\mathrm{T}}\left[\mathbb{A}, \Gamma_{\mu \nu}\right]_{\mp} \epsilon-\frac{1}{8 \sqrt{2}} \mathcal{F}^{\mu \nu} \epsilon^{\mathrm{T}}\left[\mathbb{A}, \Gamma_{\mu \nu} \Gamma_{7}\right]_{ \pm} \epsilon
\end{aligned}
$$

Here $\mathbb{A} \in \operatorname{Cliff}(6,0)$ is an arbitrary element of the Clifford algebra, while $[\cdot, \cdot]_{-}$denotes a commutator and $[\cdot, \cdot]_{+}$denotes an anti-commutator.

\section{B Differential conditions for bilinears}

We may introduce the following bilinears in the spinor $\epsilon$ :

$$
\begin{aligned}
K \equiv \epsilon^{\dagger} \Gamma_{(1)} \epsilon & =S \sin 2 \vartheta K_{1}, \\
\tilde{K} \equiv \mathrm{i} \epsilon^{\dagger} \Gamma_{(1)} \Gamma_{7} \epsilon & =-S \sin 2 \vartheta K_{2}, \\
Y \equiv \mathrm{i} \epsilon^{\dagger} \Gamma_{(2)} \epsilon & =S\left(\cos 2 \vartheta K_{1} \wedge K_{2}-J\right), \\
\tilde{Y} \equiv \mathrm{i} \epsilon^{\dagger} \Gamma_{(2)} \Gamma_{7} \epsilon & =S\left(-K_{1} \wedge K_{2}+\cos 2 \vartheta J\right), \\
Z \equiv \epsilon^{\mathrm{T}} \Gamma_{(2)} \Gamma_{7} \epsilon & =-S \sin 2 \vartheta \Omega, \\
V \equiv \mathrm{i} \epsilon^{\dagger} \Gamma_{(3)} \epsilon & =-S \sin 2 \vartheta K_{1} \wedge J, \\
\tilde{V} \equiv \epsilon^{\dagger} \Gamma_{(3)} \Gamma_{7} \epsilon & =-S \sin 2 \vartheta K_{2} \wedge J, \\
W \equiv \epsilon^{\mathrm{T}} \Gamma_{(3)} \epsilon & =S\left(-\cos 2 \vartheta K_{1}+\mathrm{i} K_{2}\right) \wedge \Omega, \\
\tilde{W} \equiv \epsilon^{\mathrm{T}} \Gamma_{(3)} \Gamma_{7} \epsilon & =S\left(K_{1}-\mathrm{i} \cos 2 \vartheta K_{2}\right) \wedge \Omega .
\end{aligned}
$$

Here $\left(K_{1}, K_{2}, J, \Omega\right)$ is the canonical $\mathrm{SU}(2)$ structure defined in section 2.2.

A straightforward but lengthy calculation shows that the Killing spinor equation (2.3) and dilatino equation (2.4) imply the following differential constraints on the bilinears in (B.1):

$$
\begin{aligned}
\mathrm{d}(X S)= & \left.\frac{\sqrt{2}}{3}\left(X^{-2} \tilde{K}-\mathrm{i} K\right\lrcorner B\right) \\
\mathrm{d}(X \tilde{S})= & \left.-\frac{1}{\sqrt{2}} K\right\lrcorner \mathcal{F}, \\
\mathrm{d}\left(X^{2} K\right)= & \left.-\frac{2 \sqrt{2}}{3} X^{-1} \tilde{Y}-\mathrm{i} X^{4} K\right\lrcorner * H-\sqrt{2} X\left(\tilde{S} \mathcal{F}-\mathrm{i} \frac{2}{3} S B\right), \\
\mathrm{d}\left(X^{-2} \tilde{K}\right)= & -\mathrm{i} K\lrcorner H \\
\mathrm{~d}\left(X^{-1} Y\right)= & \left.-\sqrt{2} \tilde{V}+\mathrm{i}(X \tilde{S}) H+\frac{1}{\sqrt{2}} X^{-2}(K\lrcorner * \mathcal{F}+\mathcal{F} \wedge \tilde{K}\right), \\
\mathrm{d}\left(X^{-1} \tilde{Y}\right)= & \left.\mathrm{i}(X S) H+\mathrm{i} \frac{\sqrt{2}}{3} X^{-2}(K\lrcorner * B+B \wedge \tilde{K}\right), \\
D\left(X^{-1} Z\right)= & -\mathrm{i} \sqrt{2} W, \\
\mathrm{~d} V= & \sqrt{2}\left(X+\frac{1}{3} X^{-3}\right) * Y+\mathrm{i} \frac{\sqrt{2}}{3} X^{-1}(\tilde{S} * B+B \wedge Y) \\
& -\frac{1}{\sqrt{2}} X^{-1}(S * \mathcal{F}+\mathcal{F} \wedge \tilde{Y})
\end{aligned}
$$




$$
\begin{aligned}
\mathrm{d} \tilde{V} & =0, \\
D W & =-\frac{1}{\sqrt{2}} X^{-1} \mathcal{F} \wedge Z, \\
D \tilde{W} & =-\sqrt{2}\left(X+\frac{1}{3} X^{-3}\right) * Z-\mathrm{i} \frac{\sqrt{2}}{3} X^{-1} B \wedge Z, \\
\mathrm{~d}\left[\left(X+\frac{1}{3} X^{-3}\right) * Y\right] & =\frac{\sqrt{2}}{3} \mathrm{i} B \wedge \tilde{V}-\frac{\mathrm{i}}{3} X^{-1} H \wedge Y+\frac{1}{3 \sqrt{2}} X^{-4}(* \mathcal{F}) \wedge \tilde{K} .
\end{aligned}
$$

Here the covariant derivatives are $D=\mathrm{d}+\mathrm{i} \mathcal{A} \wedge$, and the contraction of a $p$-form $\rho$ into a $q$-form $\lambda$ (with $q \geq p$ ) is the $(q-p)$-form $(\rho\lrcorner \lambda)_{\mu_{1} \cdots \mu_{q-p}} \equiv \frac{1}{p !} \rho^{\nu_{1} \cdots \nu_{p}} \lambda_{\nu_{1} \cdots \nu_{p}} \mu_{1} \cdots \mu_{q-p}$.

In addition to (B.2)-(B.13) it is also straightforward to show that $K$ is a Killing oneform, so that the dual vector field $\xi \equiv K^{\#}$ is a Killing vector. We may hence introduce a local coordinate $\psi$, so that $\xi=\partial_{\psi}$ and the metric is independent of $\psi$. Since $K=$ $S \sin 2 \vartheta K_{1}$, where $K_{1}$ has unit length, we may thus write

$$
K_{1}=S \sin 2 \vartheta(\mathrm{d} \psi+\sigma),
$$

where $\mathcal{L}_{\xi} \sigma=0=i_{\xi} \sigma$ and $\mathcal{L}_{\xi}(S \sin 2 \vartheta)=0$.

In order to analyse the equations (B.2)-(B.13) further we write

$$
B=B_{1} \wedge K_{1}+B_{\perp}, \quad \mathcal{F}=\mathcal{F}_{1} \wedge K_{1}+\mathcal{F}_{\perp},
$$

where $B_{1}, B_{\perp}, \mathcal{F}_{1}, \mathcal{F}_{\perp}$ are chosen to have zero contraction with $K_{1}$. The bilinear (B.2) then determines

$$
B_{1}=-\frac{3 \mathrm{i}}{\sqrt{2} S \sin 2 \vartheta} \mathrm{d}(X S)-\mathrm{i} X^{-2} K_{2} .
$$

Similarly the bilinear (B.3) is equivalent to

$$
\mathcal{F}_{1}=-\frac{\sqrt{2}}{S \sin 2 \vartheta} \mathrm{d}(X S \cos 2 \vartheta) .
$$

Contracting these last two equations with $K_{1}$, one concludes that $\mathcal{L}_{\xi}(X S)=0=\mathcal{L}_{\xi} \vartheta$. Notice also that setting $\mathbb{A}=1_{8}$ in (A.1) and taking the anti-commutator leads immediately to $\mathcal{L}_{\xi} X=0$. Having imposed (B.2), a short computation shows that equation (B.5) is equivalent to $\mathcal{L}_{\xi} B=0$. One can also deduce from (B.5) that $\mathcal{L}_{\xi} K_{2}=0$, and similarly from (B.3) it follows that $\mathcal{L}_{\xi} \mathcal{F}=0$. We may then write

$$
\mathcal{A}=-\sqrt{2} X \cot 2 \vartheta K_{1}+\mathcal{A}_{\perp} .
$$

Notice here we have made a partial gauge choice for $\mathcal{A}$. Then

$$
\mathcal{F}_{\perp}=-\sqrt{2} X S \cos 2 \vartheta \mathrm{d} \sigma+\mathrm{d} \mathcal{A}_{\perp} .
$$

Next one can show that equation (B.4) is equivalent to

$$
\begin{aligned}
X^{2} S^{2} \sin ^{2} 2 \vartheta \mathrm{d} \sigma= & \left.-\frac{2 \sqrt{2}}{3} X^{-1} S \cos 2 \vartheta J-\mathrm{i} X^{4} S \sin 2 \vartheta K_{1}\right\lrcorner * H_{\perp} \\
& +\sqrt{2} X S\left(\cos 2 \vartheta \mathcal{F}_{\perp}+\frac{2}{3} \mathrm{i} B_{\perp}\right) .
\end{aligned}
$$


Here we have defined

$$
H_{\perp} \equiv \mathrm{i}\left[\frac{3}{\sqrt{2}} \mathrm{~d}(X S)+X^{-2} S \sin 2 \vartheta K_{2}\right] \wedge \mathrm{d} \sigma+\mathrm{d} B_{\perp} .
$$

The contractions of (B.6) and (B.7) with $K_{1}$ imply that $\mathcal{L}_{\xi} J=0$. Equation (B.6) is then equivalent to

$$
\begin{aligned}
\mathrm{d}\left(X^{-1} S J\right)= & -\sqrt{2} S \sin 2 \vartheta J \wedge K_{2}-\frac{3}{2 \sqrt{2}} \cos 2 \vartheta \mathrm{d}\left[(X S)^{2} \mathrm{~d} \sigma\right]+\mathrm{i} X S \cos 2 \vartheta \mathrm{d} B_{\perp} \\
& \left.-\frac{1}{\sqrt{2}} X^{-2} S \sin 2 \vartheta\left[K_{1}\right\lrcorner * \mathcal{F}_{\perp}-K_{2} \wedge \mathcal{F}_{\perp}\right] .
\end{aligned}
$$

Similarly, one can show that (B.7) is equivalent to

$$
\begin{aligned}
\mathrm{d}\left(X^{-1} S \cos 2 \vartheta J\right)= & -\frac{3}{2 \sqrt{2}} \mathrm{~d}\left[(X S)^{2} \mathrm{~d} \sigma\right]+\mathrm{i} X S \mathrm{~d} B_{\perp} \\
& \left.+\frac{\sqrt{2}}{3} \mathrm{i} X^{-2} S \sin 2 \vartheta\left[K_{1}\right\lrcorner * B_{\perp}-K_{2} \wedge B_{\perp}\right] .
\end{aligned}
$$

The contraction of equation (B.8) with $K_{1}$, in the gauge in which $\mathcal{A}$ is given by (B.18), simply gives $\mathcal{L}_{\xi} \Omega=0$. Equation (B.8) is then equivalent to

$$
D_{\perp}\left(X^{-1} S \sin 2 \vartheta \Omega\right)=-\sqrt{2} S \Omega \wedge K_{2},
$$

where $D_{\perp} \equiv \mathrm{d}+\mathrm{i} \mathcal{A}_{\perp} \wedge$.

Finally we move onto the three-form bilinears. Equation (B.10) states

$$
\mathrm{d}\left(S \sin 2 \vartheta J \wedge K_{2}\right)=0 .
$$

The contraction of $K_{1}$ into (B.11) is equivalent to (B.24), while the remainder of this equation turns out to be the integrability condition for (B.24). Next one can show that $K_{1}$ contracted into (B.9) is implied by (B.22) and (B.23), while the remainder of this equation reads

$$
\begin{aligned}
-S^{2} \sin ^{2} 2 \vartheta J \wedge \mathrm{d} \sigma= & \left.\sqrt{2} S \cos 2 \vartheta\left(X+\frac{2}{3} X^{-3}\right) \frac{1}{2} J \wedge J-2 S K_{1}\right\lrcorner * \mathrm{~d} \vartheta \\
& -\frac{1}{\sqrt{2}} X^{-1} S J \wedge\left(\cos 2 \vartheta \mathcal{F}_{\perp}+\frac{2}{3} \mathrm{i} B_{\perp}\right) .
\end{aligned}
$$

Next we find that $K_{1}$ contracted into (B.12) is implied by (B.24). Using (B.24) the remainder of this equation reads

$$
S^{2} \sin ^{2} 2 \vartheta \Omega \wedge \mathrm{d} \sigma=-2 \mathrm{i} S \mathrm{~d} \vartheta \wedge K_{2} \wedge \Omega+\frac{1}{\sqrt{2}} X^{-1} S \Omega \wedge\left(\cos 2 \vartheta \mathcal{F}_{\perp}+\frac{2}{3} \mathrm{i} B_{\perp}\right) .
$$

The contraction of $K_{1}$ into (B.13) can again be shown to follow from equations derived so far, while the remaining content of this equation is (on using various other equations) equivalent to

$$
\left.\left.X^{4} K_{2}\right\lrcorner \mathrm{~d}\left(X^{-3} S \sin 2 \vartheta\right)+\sqrt{2} S\left(X^{2}-\frac{2}{3} X^{-2}\right)+\frac{1}{\sqrt{2}} S J\right\lrcorner\left(\mathcal{F}_{\perp}+\frac{2}{3} \mathrm{i} \cos 2 \vartheta B_{\perp}\right)=0 .
$$




\section{More on the dilatino equation}

In the Abelian case of interest, the dilatino equation (2.4) may be written as $\delta \chi=0$, where we have introduced

$$
\begin{aligned}
\delta \chi \equiv & -\mathrm{i} X^{-1} \partial_{\mu} X \Gamma^{\mu} \epsilon+\frac{1}{2 \sqrt{2}}\left(X-X^{-3}\right) \Gamma_{7} \epsilon+\frac{\mathrm{i}}{24} X^{2} H_{\mu \nu \rho} \Gamma^{\mu \nu \rho} \Gamma_{7} \epsilon \\
& -\frac{1}{12 \sqrt{2}} X^{-1} B_{\mu \nu} \Gamma^{\mu \nu} \epsilon-\frac{\mathrm{i}}{8 \sqrt{2}} X^{-1} \mathcal{F}_{\mu \nu} \Gamma^{\mu \nu} \Gamma_{7} \epsilon .
\end{aligned}
$$

Recall here that $A_{\mu}^{1}=A_{\mu}^{2}=0$, while $\mathcal{A}_{\mu} \equiv A_{\mu}^{3}$, with curvature $\mathcal{F}=\mathrm{d} \mathcal{A}$. The right hand side of (C.1) is an 8-component spinor, and thus $\delta \chi=0$ comprise 8 algebraic equations for $\epsilon=\epsilon_{+}+\epsilon_{-}$.

We begin by noting that neither of the definite chirality projections $\epsilon_{+}$nor $\epsilon_{-}$can be identically zero. For if $\epsilon_{ \pm}=0$, respectively, then we in fact have an $\mathrm{SU}(3)$ structure, rather than $\mathrm{SU}(2)$ structure, and the bilinear $W \equiv \epsilon^{\mathrm{T}} \Gamma_{(3)} \epsilon=\Omega_{\mp}$ is the corresponding complex three-form. However, since the left hand side of equation (B.8) of appendix B is identically zero in this case, we would deduce that $\Omega_{\mp}=0$ and hence $\epsilon_{\mp}=0$.

On an open dense subset where $\epsilon_{ \pm}$are both non-zero, we then have that $\left\{\epsilon_{ \pm}, \Gamma_{\mu} \epsilon_{ \pm}^{*}\right\}$ span the positive and negative chirality spin bundles $\mathcal{S}^{ \pm}$, respectively. Recall from (2.8) that $\epsilon_{+}=\sqrt{S} \cos \vartheta \eta_{1}, \epsilon_{-}=\sqrt{S} \sin \vartheta \eta_{2}^{*}$, where $\eta_{1}$ and $\eta_{2}$ have unit norm. In an orthonormal frame $\left(e^{1}, \ldots, e^{4}, e^{5} \equiv K_{1}, e^{6} \equiv K_{2}\right)$ in which the canonical SU(2) structure defined by $\eta_{1}$ and $\eta_{2}$ is given by $(2.21)$, one can easily check that $\left\{\epsilon_{+}, \Gamma_{1} \epsilon_{+}^{*}, \Gamma_{3} \epsilon_{+}^{*}, \Gamma_{5} \epsilon_{+}^{*}\right\}$ form a basis for $\mathcal{S}^{+}$, while $\left\{\epsilon_{-}, \Gamma_{1} \epsilon_{-}^{*}, \Gamma_{3} \epsilon_{-}^{*}, \Gamma_{5} \epsilon_{-}^{*}\right\}$ form a basis for $\mathcal{S}^{-}$. Thus in order for the dilatino equation $\delta \chi=0$ to hold, it is sufficient to check that the contraction of (C.1) with $\epsilon_{ \pm}^{\dagger}$ and $\epsilon_{ \pm}^{\mathrm{T}} \Gamma_{\mu}$ is zero. These are equivalent to two scalar and two one-form equations, respectively, that may be expressed in terms of the bilinears (B.1). Specifically, we may take the two scalar contractions to be

$$
\begin{aligned}
\epsilon^{\dagger} \delta \chi= & -\mathrm{i} X^{-1} \partial_{\mu} X K^{\mu}+\frac{1}{2 \sqrt{2}}\left(X-X^{-3}\right) \tilde{S}+\frac{\mathrm{i}}{24} X^{2} H_{\mu \nu \rho} \tilde{V}^{\mu \nu \rho} \\
& +\frac{\mathrm{i}}{12 \sqrt{2}} X^{-1} B_{\mu \nu} Y^{\mu \nu}-\frac{1}{8 \sqrt{2}} X^{-1} \mathcal{F}_{\mu \nu} \tilde{Y}^{\mu \nu}, \\
\epsilon^{\dagger} \Gamma_{7} \delta \chi= & X^{-1} \partial_{\mu} X \tilde{K}^{\mu}+\frac{1}{2 \sqrt{2}}\left(X-X^{-3}\right) S-\frac{1}{24} X^{2} H_{\mu \nu \rho} V^{\mu \nu \rho} \\
& +\frac{\mathrm{i}}{12 \sqrt{2}} X^{-1} B_{\mu \nu} \tilde{Y}^{\mu \nu}-\frac{1}{8 \sqrt{2}} X^{-1} \mathcal{F}_{\mu \nu} Y^{\mu \nu},
\end{aligned}
$$

while the two one-form contractions are

$$
\begin{aligned}
\epsilon^{\mathrm{T}} \Gamma_{\sigma} \delta \chi= & \frac{\mathrm{i}}{8} X^{2} H_{\mu \nu \sigma} Z^{\mu \nu}-\frac{1}{12 \sqrt{2}} X^{-1} B^{\mu \nu} W_{\mu \nu \sigma}-\frac{\mathrm{i}}{8 \sqrt{2}} X^{-1} \mathcal{F}^{\mu \nu} \tilde{W}_{\mu \nu \sigma}, \\
\epsilon^{\mathrm{T}} \Gamma_{\sigma} \Gamma_{7} \delta \chi= & -\mathrm{i} X^{-1} \partial^{\mu} X Z_{\mu \sigma}-\frac{1}{8} X^{2}(* H)_{\mu \nu \sigma} Z^{\mu \nu} \\
& -\frac{1}{12 \sqrt{2}} X^{-1} B^{\mu \nu} \tilde{W}_{\mu \nu \sigma}-\frac{\mathrm{i}}{8 \sqrt{2}} X^{-1} \mathcal{F}^{\mu \nu} W_{\mu \nu \sigma} .
\end{aligned}
$$

The dilatino equation $\delta \chi=0$ is thus equivalent to the the right hand sides of (C.2) and (C.3) being zero. A tedious, but straightforward, calculation shows that $\delta \chi=0$ is implied by the differential constraints (2.18). 


\section{Integrability conditions}

For what follows it will be convenient to record the component form of the Romans field equations in (2.1) and (2.2):

$$
\begin{aligned}
\left(\mathscr{E}_{g}\right)_{\mu \nu} \equiv & R_{\mu \nu}-4 X^{-2} \partial_{\mu} X \partial_{\nu} X-\left(\frac{1}{18} X^{-6}-\frac{1}{2} X^{2}-\frac{2}{3} X^{-2}\right) g_{\mu \nu} \\
& -\frac{1}{4} X^{4}\left(H_{\mu}{ }^{\rho \sigma} H_{\nu \rho \sigma}-\frac{1}{6} g_{\mu \nu} H^{\rho \sigma \tau} H_{\rho \sigma \tau}\right)-\frac{2}{9} X^{-2}\left(B_{\mu}{ }^{\rho} B_{\nu \rho}-\frac{1}{8} g_{\mu \nu} B^{\rho \sigma} B_{\rho \sigma}\right) \\
& -\frac{1}{2} X^{-2}\left(F_{\mu}^{i \rho} F_{\nu \rho}^{i}-\frac{1}{8} g_{\mu \nu} F^{i \rho \sigma} F_{\rho \sigma}^{i}\right), \\
\left(\mathscr{E}_{X}\right) \equiv & \nabla^{\mu}\left(X^{-1} \partial_{\mu} X\right)+\left(\frac{1}{2} X^{2}-\frac{2}{3} X^{-2}+\frac{1}{6} X^{-6}\right)-\frac{1}{24} X^{4} H^{\mu \nu \rho} H_{\mu \nu \rho} \\
& +\frac{1}{16} X^{-2}\left(\frac{4}{9} B^{\mu \nu} B_{\mu \nu}+F^{i \mu \nu} F_{\mu \nu}^{i}\right), \\
\left(\mathscr{E}_{A}\right)^{\mu} \equiv & \nabla_{\nu}\left(X^{-2} B^{\nu \mu}\right)-\frac{\mathrm{i}}{12} \varepsilon^{\mu \nu \rho \sigma \tau \kappa} B_{\nu \rho} H_{\sigma \tau \kappa}, \\
\left(\mathscr{E}_{A^{i}}\right)^{\mu} \equiv & D_{\nu}\left(X^{-2} F^{i \nu \mu}\right)-\frac{\mathrm{i}}{12} \varepsilon^{\mu \nu \rho \sigma \tau \kappa} F_{\nu \rho}^{i} H_{\sigma \tau \kappa}, \\
\left(\mathscr{E}_{B}\right)^{\mu \nu} \equiv & \nabla_{\rho}\left(X^{4} H^{\rho \mu \nu}\right)-\frac{4}{9} X^{-2} B^{\mu \nu}-\frac{\mathrm{i}}{8} \varepsilon^{\mu \nu \rho \sigma \tau \kappa}\left(\frac{4}{9} B_{\rho \sigma} B_{\tau \kappa}+F_{\rho \sigma}^{i} F_{\tau \kappa}^{i}\right) .
\end{aligned}
$$

The equations of motion are then $\mathscr{E}_{\text {field }}=0$. The field $A$ is the Stueckelberg one-form, that we set to zero using the gauge symmetry of the theory. Its equation of motion $\mathscr{E}_{A}=0$ follows from taking the divergence of the $B$-field equation of motion $\mathscr{E}_{B}=0$. We also introduce

$$
\begin{aligned}
\left(\mathscr{B}_{F}\right)_{\mu \nu \rho} & \equiv \nabla_{[\mu} B_{\nu \rho]}-\frac{1}{3} H_{\mu \nu \rho} \\
\left(\mathscr{B}_{F^{i}}\right)_{\mu \nu \rho} & \equiv D_{[\mu} F_{\nu \rho]}^{i} \\
\left(\mathscr{B}_{H}\right)_{\mu \nu \rho \sigma} & \equiv \nabla_{[\mu} H_{\nu \rho \sigma]}
\end{aligned}
$$

Note that $\mathscr{B}_{\text {field }}$ vanish automatically as a consequence of the Bianchi identities. For the Abelian case studied in the main text recall that $F_{\mu \nu}^{1}=F_{\mu \nu}^{2}=0$ while $\mathcal{F}_{\mu \nu} \equiv F_{\mu \nu}^{3}$.

In what follows we will show that supersymmetry together with $\left(\mathscr{E}_{B}\right)_{\perp}=0$ imply the equations of motion for all the fields. We begin by taking the exterior derivative of (B.4) to obtain

$$
\left.0=-\frac{2 \sqrt{2}}{3} \mathrm{~d}\left(X^{-1} \tilde{Y}\right)-\mathrm{id}\left(X^{4} K\right\lrcorner * H\right)+\frac{2 \sqrt{2}}{3} \mathrm{id}[X S B]-\sqrt{2} \mathcal{F} \wedge \mathrm{d}(X \tilde{S}) .
$$

Using (B.2), (B.3) and (B.7) then gives

$$
\left.\left.\left.\left.\left.0=-\mathrm{id}\left(X^{4} K\right\lrcorner * H\right)\right)-\frac{4}{9} \mathrm{i} K\right\lrcorner * B+\frac{4}{9} B \wedge(K\lrcorner B\right)+\mathcal{F} \wedge(K\lrcorner \mathcal{F}\right) .
$$

Since $\mathcal{L}_{\xi}\left(X^{4} * H\right)=0$ it hence follows that $\left.K_{1}\right\lrcorner \mathscr{E}_{B}=0$. Recall that

$$
\left.\mathscr{E}_{B}=K_{1} \wedge\left(K_{1}\right\lrcorner \mathscr{E}_{B}\right)+\left(\mathscr{E}_{B}\right)_{\perp}
$$


In general it is not true that supersymmetry implies $\left(\mathscr{E}_{B}\right)_{\perp}=0$. We henceforth impose this equation, and continue our analysis by taking the exterior derivative of (B.13). After a computation we find this implies

$$
\frac{2}{3} \mathrm{i}\left[\mathrm{d}\left(X^{-2} * B\right)+\mathrm{i} B \wedge H\right](X \tilde{S})-\left[\mathrm{d}\left(X^{-2} * \mathcal{F}\right)+\mathrm{i} \mathcal{F} \wedge H\right](X S)=0 .
$$

Since $\mathscr{E}_{B}=0$ implies $\mathscr{E}_{A}=0$, (D.6) implies $\mathscr{E}_{\mathcal{A}}=0$.

To obtain the remaining equations of motion, we may use the integrability conditions for the dilatino equation (2.4) and Killing spinor equation (2.3) derived in [19]:

$$
\begin{aligned}
0= & \mathrm{i}\left(\mathscr{E}_{X}\right) \epsilon_{I}-\frac{1}{6 \sqrt{2}} X\left(\mathscr{E}_{A}\right)_{\mu} \Gamma^{\mu} \epsilon_{I}-\frac{\mathrm{i}}{4 \sqrt{2}} X\left(\mathscr{E}_{A^{i}}\right)_{\mu} \Gamma^{\mu} \Gamma_{7}\left(\sigma_{i}\right)_{I}^{J} \epsilon_{J}+\frac{\mathrm{i}}{8} X^{-2}\left(\mathscr{E}_{B}\right)_{\mu \nu} \Gamma^{\mu \nu} \Gamma_{7} \epsilon_{I} \\
& -\frac{1}{12 \sqrt{2}} X^{-1}\left(\mathscr{B}_{F}\right)_{\mu \nu \rho} \Gamma^{\mu \nu \rho} \epsilon_{I}-\frac{\mathrm{i}}{8 \sqrt{2}} X^{-1}\left(\mathscr{B}_{F^{i}}\right)_{\mu \nu \rho} \Gamma^{\mu \nu \rho} \Gamma_{7}\left(\sigma_{i}\right)_{I}^{J} \epsilon_{J} \\
& +\frac{\mathrm{i}}{24} X^{2}\left(\mathscr{B}_{H}\right)_{\mu \nu \rho \sigma} \Gamma^{\mu \nu \rho \sigma} \Gamma_{7} \epsilon_{I}, \\
0= & \frac{1}{2}\left(\mathscr{E}_{X}\right) \Gamma_{\mu} \epsilon_{I}-\frac{1}{2}\left(\mathscr{E}_{g}\right)_{\mu \nu} \Gamma^{\nu} \epsilon_{I}-\frac{1}{8} X^{-2}\left(\mathscr{E}_{B}\right)^{\nu \rho} \Gamma_{\mu \nu \rho} \Gamma_{7} \epsilon_{I} \\
& -\frac{\mathrm{i}}{3 \sqrt{2}} X\left(\mathscr{E}_{A}\right)_{\mu} \epsilon_{I}+\frac{1}{2 \sqrt{2}} X\left(\mathscr{E}_{A^{i}}\right)_{\mu} \Gamma_{7}\left(\sigma_{i}\right)_{I}^{J} \epsilon_{J}-\frac{1}{24} X^{2}\left(\mathscr{B}_{H}\right)^{\nu \rho \sigma \tau} \Gamma_{\mu \nu \rho \sigma \tau} \Gamma_{7} \epsilon_{I} \\
& -\frac{\mathrm{i}}{2 \sqrt{2}} X^{-1}\left(\mathscr{B}_{F}\right)_{\mu \nu \rho} \Gamma^{\nu \rho} \epsilon_{I}+\frac{3}{4 \sqrt{2}} X^{-1}\left(\mathscr{B}_{F^{i}}\right)_{\mu \nu \rho} \Gamma^{\nu \rho} \Gamma_{7}\left(\sigma_{i}\right)_{I}^{J} \epsilon_{J} .
\end{aligned}
$$

Since $\mathscr{B}_{\text {field }}=0$, and given the results above, (D.7) immediately implies $\mathscr{E}_{X}=0$. Using this, and contracting (D.8) with $\epsilon^{\dagger} \Gamma^{v}$, we deduce the Einstein equation $\mathscr{E}_{g}=0$.

\section{E Supersymmetry of the fundamental string}

In this appendix we show that the fundamental string considered in section 3.2 is supersymmetric.

As explained in [25] and [15], BPS Wilson loops in the fundamental representation are dual to fundamental strings in the massive type IIA background $M_{6} \times S^{4}$. More precisely the string sits at the "north pole" of the four-sphere and wraps the $K_{1}-K_{2}$ direction of the SU(2) structure on $M_{6}$. Since the dual vector field to $K_{1}$ is proportional to the supersymmetric Killing vector $\xi$, this means that the dual Wilson loop on the conformal boundary of $M_{6}$ wraps an orbit of $\xi$, as expected from supersymmetry. It then remains to show that the fundamental string is itself supersymmetric. This amounts to a certain projection condition on the ten-dimensional Killing spinor in massive IIA. Following a similar computation to [25], one can show this reduces to the following projection condition on the six-dimensional spinor $\epsilon$ on $M_{6}$ :

$$
\left(1+\mathrm{i} \Gamma_{7} \Gamma_{56}\right) \epsilon=0 .
$$

Here recall that the orthonormal frame components are $e^{5}=K_{1}$ and $e^{6}=K_{2}$. Recall also from section 2.2 that $\epsilon=\epsilon_{+}+\epsilon_{-}$, where

$$
\epsilon_{+}=\sqrt{S} \cos \vartheta \eta_{1}, \quad \epsilon_{-}=\sqrt{S} \sin \vartheta \eta_{2}^{*}
$$


The projection conditions [21]

$$
\Gamma_{7} \eta_{1}=-\eta_{1}, \quad \Gamma_{7} \eta_{2}^{*}=\eta_{2}^{*}, \quad-\Gamma_{56} \eta_{1}=\mathrm{i} \eta_{1}, \quad-\Gamma_{56} \eta_{2}=\mathrm{i} \eta_{2},
$$

together with the fact that the Cliff $(6,0)$ matrices are purely imaginary then immediately imply that (E.1) is indeed satisfied. Consequently the fundamental string wrapping the $K_{1}-K_{2}$ direction, at the north pole of the internal $S^{4}$, is indeed supersymmetric.

Open Access. This article is distributed under the terms of the Creative Commons Attribution License (CC-BY 4.0), which permits any use, distribution and reproduction in any medium, provided the original author(s) and source are credited.

\section{References}

[1] L.F. Alday, D. Martelli, P. Richmond and J. Sparks, Localization on three-manifolds, JHEP 10 (2013) 095 [arXiv: 1307.6848] [INSPIRE].

[2] C. Closset, T.T. Dumitrescu, G. Festuccia and Z. Komargodski, The geometry of supersymmetric partition functions, JHEP 01 (2014) 124 [arXiv: 1309.5876] [INSPIRE].

[3] J. Källén and M. Zabzine, Twisted supersymmetric 5D Yang-Mills theory and contact geometry, JHEP 05 (2012) 125 [arXiv:1202.1956] [INSPIRE].

[4] K. Hosomichi, R.-K. Seong and S. Terashima, Supersymmetric gauge theories on the five-sphere, Nucl. Phys. B 865 (2012) 376 [arXiv:1203.0371] [InSPIRE].

[5] J. Källén, J. Qiu and M. Zabzine, The perturbative partition function of supersymmetric $5 D$ Yang-Mills theory with matter on the five-sphere, JHEP 08 (2012) 157 [arXiv:1206.6008] [INSPIRE].

[6] H.-C. Kim and S. Kim, M5-branes from gauge theories on the 5-sphere, JHEP 05 (2013) 144 [arXiv: 1206.6339] [INSPIRE].

[7] Y. Imamura, Supersymmetric theories on squashed five-sphere, PTEP 2013 (2013) 013B04 [arXiv: 1209.0561] [INSPIRE].

[8] Y. Imamura, Perturbative partition function for squashed $S^{5}$, arXiv:1210.6308 [INSPIRE].

[9] J. Qiu and M. Zabzine, 5D super Yang-Mills on $Y^{p, q}$ Sasaki-Einstein manifolds, Commun. Math. Phys. 333 (2015) 861 [arXiv:1307.3149] [INSPIRE].

[10] J. Qiu and M. Zabzine, Factorization of 5D super Yang-Mills theory on $Y^{p, q}$ spaces, Phys. Rev. D 89 (2014) 065040 [arXiv: 1312.3475] [INSPIRE].

[11] J. Schmude, Localisation on Sasaki-Einstein manifolds from holomorphic functions on the cone, JHEP 01 (2015) 119 [arXiv:1401.3266] [INSPIRE].

[12] Y. Pan, Rigid supersymmetry on 5-dimensional Riemannian manifolds and contact geometry, JHEP 05 (2014) 041 [arXiv: 1308.1567] [INSPIRE].

[13] Y. Imamura and H. Matsuno, Supersymmetric backgrounds from $5 d N=1$ supergravity, JHEP 07 (2014) 055 [arXiv: 1404.0210] [inSPIRE].

[14] Y. Pan, 5D Higgs branch localization, Seiberg-Witten equations and contact geometry, JHEP 01 (2015) 145 [arXiv: 1406.5236] [INSPIRE]. 
[15] L.F. Alday, P.B. Genolini, M. Fluder, P. Richmond and J. Sparks, Supersymmetric gauge theories on five-manifolds, JHEP 08 (2015) 007 [arXiv: 1503.09090] [INSPIRE].

[16] Y. Pan and J. Schmude, On rigid supersymmetry and notions of holomorphy in five dimensions, JHEP 11 (2015) 041 [arXiv: 1504.00321] [INSPIRE].

[17] A. Pini, D. Rodriguez-Gomez and J. Schmude, Rigid supersymmetry from conformal supergravity in five dimensions, JHEP 09 (2015) 118 [arXiv: 1504.04340] [INSPIRE].

[18] L.F. Alday, M. Fluder, P. Richmond and J. Sparks, Gravity dual of supersymmetric gauge theories on a squashed five-sphere, Phys. Rev. Lett. 113 (2014) 141601 [arXiv:1404.1925] [INSPIRE].

[19] L.F. Alday, M. Fluder, C.M. Gregory, P. Richmond and J. Sparks, Supersymmetric gauge theories on squashed five-spheres and their gravity duals, JHEP 09 (2014) 067 [arXiv: 1405.7194] [INSPIRE].

[20] L.J. Romans, The F(4) gauged supergravity in six-dimensions, Nucl. Phys. B 269 (1986) 691 [INSPIRE].

[21] J.P. Gauntlett, D. Martelli, J. Sparks and D. Waldram, Supersymmetric AdS $S_{5}$ solutions of M-theory, Class. Quant. Grav. 21 (2004) 4335 [hep-th/0402153] [INSPIRE].

[22] C. Klare, A. Tomasiello and A. Zaffaroni, Supersymmetry on curved spaces and holography, JHEP 08 (2012) 061 [arXiv: 1205.1062] [inSPIRE].

[23] C. Klare and A. Zaffaroni, Extended supersymmetry on curved spaces, JHEP 10 (2013) 218 [arXiv: 1308.1102] [INSPIRE].

[24] K. Hristov, A. Tomasiello and A. Zaffaroni, Supersymmetry on three-dimensional Lorentzian curved spaces and black hole holography, JHEP 05 (2013) 057 [arXiv:1302.5228] [INSPIRE].

[25] B. Assel, J. Estes and M. Yamazaki, Wilson Loops in $5 D N=1$ SCFTs and AdS/CFT, Annales Henri Poincaré 15 (2014) 589 [arXiv: 1212.1202] [INSPIRE].

[26] D.L. Jafferis and S.S. Pufu, Exact results for five-dimensional superconformal field theories with gravity duals, JHEP 05 (2014) 032 [arXiv:1207.4359] [INSPIRE].

[27] M. Cvetič, H. Lü and C.N. Pope, Gauged six-dimensional supergravity from massive type IIA, Phys. Rev. Lett. 83 (1999) 5226 [hep-th/9906221] [INSPIRE].

[28] J. Qiu, L. Tizzano, J. Winding and M. Zabzine, Gluing Nekrasov partition functions, Commun. Math. Phys. 337 (2015) 785 [arXiv:1403.2945] [INSPIRE].

[29] S. Ferrara, A. Kehagias, H. Partouche and A. Zaffaroni, AdS $S_{6}$ interpretation of $5 D$ superconformal field theories, Phys. Lett. B 431 (1998) 57 [hep-th/9804006] [INSPIRE].

[30] A. Brandhuber and Y. Oz, The D4-D8 brane system and five-dimensional fixed points, Phys. Lett. B 460 (1999) 307 [hep-th/9905148] [INSPIRE]. 